\title{
Stability of stationary solutions to the outflow problem for full compressible Navier-Stokes equations with large initial perturbation
}

\author{
Ling Wan ${ }^{\mathrm{a}}$, Tao Wang ${ }^{\mathrm{a}, *}$, Qingyang Zou ${ }^{\mathrm{b}}$ \\ March 28, 2021 \\ a School of Mathematics and Statistics, Wuhan University, Wuhan 430072, China \\ b School of Science, Wuhan University of Science and Technology, Wuhan 430081, China
}

\begin{abstract}
We investigate the large-time behavior of solutions to an outflow problem of the full compressible Navier-Stokes equations in the half line. The non-degenerate stationary solution is shown to be asymptotically stable under large initial perturbation with no restriction on the adiabatic exponent $\gamma$, provided that the boundary strength is sufficiently small. The proofs are based on the standard energy method and the crucial step is to obtain positive lower and upper bounds of the density and the temperature uniformly in time and space.
\end{abstract}

Keywords: full compressible Navier-Stokes equations; outflow problem; stationary solution; stability; large initial perturbation

Mathematics Subject Classification: 76N10 (35B35 35B40 35Q35)

\section{Introduction}

We study an initial-boundary value problem for the full compressible Navier-Stokes equations

$$
\left\{\begin{aligned}
\rho_{t}+(\rho u)_{x} & =0 \\
(\rho u)_{t}+\left(\rho u^{2}+P\right)_{x} & =\left(\mu u_{x}\right)_{x} \\
(\rho E)_{t}+(\rho u E+u P)_{x} & =\left(\kappa \theta_{x}+\mu u u_{x}\right)_{x}
\end{aligned}\right.
$$

where $t>0$ is the time variable, $x \in \mathbb{R}_{+}:=(0, \infty)$ is the spatial variable, and the primary dependent variables are the density $\rho$, fluid velocity $u$ and absolute temperature $\theta$. The specific total energy $E=$ $e+\frac{1}{2} u^{2}$ with $e$ being the specific internal energy. The viscosity coefficient $\mu$ and the heat conductivity $\kappa$ are assumed to be positive constants. Here we focus on the ideal polytropic gas, that is, the pressure $P$ and the specific internal energy $e$ are given by the constitutive relations

$$
P=R \rho \theta, \quad e=c_{v} \theta,
$$

where $R>0$ is the gas constant and the specific heat $c_{v}=R /(\gamma-1)$ with $\gamma>1$ being the adiabatic exponent.

The system (1.1) is supplemented with the initial data

$$
\left.(\rho, u, \theta)\right|_{t=0}=\left(\rho_{0}, u_{0}, \theta_{0}\right),
$$

${ }^{*}$ Corresponding author. E-mail address: tao.wang@whu.edu.cn 
which are assumed to satisfy

$$
\lim _{x \rightarrow \infty}\left(\rho_{0}, u_{0}, \theta_{0}\right)(x)=\left(\rho_{+}, u_{+}, \theta_{+}\right),
$$

where $\rho_{+}>0, u_{+}$and $\theta_{+}>0$ are constants. For boundary conditions, we take

$$
u(t, 0)=u_{-}, \quad \theta(t, 0)=\theta_{-}>0,
$$

where $u_{-}<0$ and $\theta_{-}$are constants.

The assumption $u_{-}<0$ means that the fluid blows out from the boundary, and hence the problem (1.1)-(1.5) is called the outflow problem. In the case of $u_{-}=0$ the problem is called the impermeable wall problem, while the problem (1.1)-(1.5) with the additional boundary condition $\rho(t, 0)=\rho_{-}$in the case of $u_{-}>0$ is called the inflow problem.

Matsumura [15] considered initial-boundary value problems for the isentropic Navier-Stokes equations in the half line $\mathbb{R}_{+}$and proposed a complete classification about the precise description of the largetime behaviors of solutions. Since then, some results have been obtained for the rigorous mathematical justification of this classification. For the impermeable wall problem, the stability for the viscous shock wave is obtained in [16] for small initial perturbation, while Matsumura-Nishihara [17] showed that the rarefaction wave is asymptotically stable under large initial perturbation. For the inflow problem under small initial perturbation, see [18] for the stability of the boundary layer solution and its superposition with the rarefaction wave, and see [3] for the stability of the viscous shock wave and its superposition with the boundary layer solution. Recently, Fan et al. [1] established the asymptotic stability of both the boundary layer solution and the supersonic rarefaction wave for a certain class of large initial perturbation. For the outflow problem, the stability of the stationary solution and its superposition with the rarefaction wave is proved in $[9,10]$ under small initial perturbation, while a convergence rate of solutions toward the stationary solution was obtained in [19] provided that the initial perturbation belongs to some weighted Sobolev space. Huang-Qin [5] improved the results in [9, 10] to large initial perturbation.

There have been some works on the large-time behaviour of solutions to the initial-boundary value problems of the full compressible Navier-Stokes equations (1.1)-(1.2) in the half line. For the inflow problem, Qin-Wang [23, 24] showed the existence of the boundary layer solution as well as the stability of the boundary layer and its superposition with the viscous contact wave and rarefaction waves. The asymptotic stability of the rarefaction wave, boundary layer solution, and their superposition is proved in [2] for both the impermeable wall problem and the inflow problem. Kawashima et al. [8] proved the existence, the nonlinear stability and the convergence rate of the stationary solution for the outflow problem. We note that the results in [2, 8, 23, 24] are all concerned with small initial perturbation. Thus a problem of interest is whether stability results on the stationary solution and the rarefaction wave hold for the outflow problem (1.1)-(1.5) with large initial perturbation as in [5] for the isentropic model. In this direction, motivated by [21], Qin [22] proved that the non-degenerate stationary solution is asymptotically stable under "partially" large initial perturbation with the technical condition that the adiabatic exponent $\gamma$ is close to 1 .

In this article, we establish the large-time behavior of solutions toward stationary solutions to the outflow problem (1.1)-(1.5) under large initial perturbation without any restriction on the adiabatic exponent $\gamma$. We expect that the solution converges to a stationary solution $(\tilde{\rho}, \tilde{u}, \tilde{\theta})(x)$ of $(1.1)$ :

$$
\left\{\begin{aligned}
(\tilde{\rho} \tilde{u})^{\prime} & =0, \\
\left(\tilde{\rho} \tilde{u}^{2}+\tilde{P}\right)^{\prime} & =\mu \tilde{u}^{\prime \prime}, \\
(\tilde{\rho} \tilde{u} \tilde{E}+\tilde{u} \tilde{P})^{\prime} & =\kappa \tilde{\theta}^{\prime \prime}+\mu\left(\tilde{u} \tilde{u}^{\prime}\right)^{\prime},
\end{aligned}\right.
$$

where $\tilde{P}:=R \tilde{\rho} \tilde{\theta}$ and $\tilde{E}:=c_{v} \tilde{\theta}+\frac{1}{2} \tilde{u}^{2}$. We also assume that the stationary solution satisfies the far-field condition (1.4) and the boundary condition (1.5):

$$
(\tilde{u}, \tilde{\theta})(0)=\left(u_{-}, \theta_{-}\right), \quad \lim _{x \rightarrow \infty}(\tilde{\rho}, \tilde{u}, \tilde{\theta})(x)=\left(\rho_{+}, u_{+}, \theta_{+}\right)
$$

We define the boundary strength $\delta$ as

$$
\delta:=\left|\left(u_{+}-u_{-}, \theta_{+}-\theta_{-}\right)\right|,
$$


and the Mach number at infinity as

$$
M_{+}:=\frac{\left|u_{+}\right|}{c_{+}}
$$

where $c_{+}:=\sqrt{R \gamma \theta_{+}}$is the sound speed. The existence and the properties of the stationary solution $(\tilde{\rho}, \tilde{u}, \tilde{\theta})$ satisfying (1.6) and (1.7) are quoted in the following lemma.

Lemma 1.1 (Existence of stationary solution [8]). Suppose that $\left(u_{-}, \theta_{-}\right)$satisfies

$$
\left(u_{-}, \theta_{-}\right) \in \mathcal{M}^{+}:=\left\{(u, \theta) \in \mathbb{R}^{2}:\left|\left(u-u_{+}, \theta-\theta_{+}\right)\right|<\delta_{0}\right\}
$$

for a certain positive constant $\delta_{0}$.

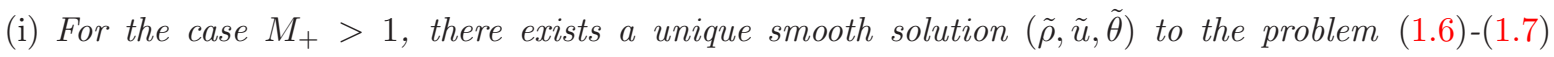
satisfying

$$
\left|\partial_{x}^{n}\left(\tilde{\rho}-\rho_{+}, \tilde{u}-u_{+}, \tilde{\theta}-\theta_{+}\right)(x)\right| \leq C \delta e^{-c x}
$$

for integer $n=0,1,2, \cdots$, where $C$ and $c$ are positive constants.

(ii) For the case $M_{+}=1$, there exists a certain region $\mathcal{M}^{0} \subset \mathcal{M}^{+}$such that if $\left(u_{-}, \theta_{-}\right) \in \mathcal{M}^{0}$, then there exists a unique smooth solution $(\tilde{\rho}, \tilde{u}, \tilde{\theta})$ to (1.6)-(1.7) satisfying

$$
\left|\partial_{x}^{n}\left(\tilde{\rho}-\rho_{+}, \tilde{u}-u_{+}, \tilde{\theta}-\theta_{+}\right)(x)\right| \leq \frac{C \delta^{n+1}}{(1+\delta x)^{n+1}}+C \delta e^{-c x}
$$

for integer $n=0,1,2, \cdots$.

(iii) For the case $M_{+}<1$, there exists a curve $\mathcal{M}^{-} \subset \mathcal{M}^{+}$such that if $\left(u_{-}, \theta_{-}\right) \in \mathcal{M}^{+}$, then there exists a unique smooth solution $(\tilde{\rho}, \tilde{u}, \tilde{\theta})$ to the problem (1.6)-(1.7) satisfying (1.9).

Our main result is now stated as follows.

Theorem 1. Assume that there exists an stationary solution $(\tilde{\rho}, \tilde{u}, \tilde{\theta})$ to the problem (1.6)-(1.7) satisfying (1.9). If

$$
\inf _{x \in \mathbb{R}_{+}}\left(\rho_{0}, \theta_{0}\right)(x)>0, \quad\left(\rho_{0}-\tilde{\rho}, u_{0}-\tilde{u}, \theta_{0}-\tilde{\theta}\right) \in H^{1}\left(\mathbb{R}_{+}\right),
$$

then there is a positive constant $\epsilon_{1}$ such that if $\delta \leq \epsilon_{1}$, then the outflow problem (1.1)-(1.5) admits a unique solution $(\rho, u, \theta)$ satisfying

$$
\begin{gathered}
(\rho-\tilde{\rho}, u-\tilde{u}, \theta-\tilde{\theta}) \in C\left([0, \infty) ; H^{1}\left(\mathbb{R}_{+}\right)\right), \\
\rho_{x}-\tilde{\rho}_{x} \in L^{2}\left(0, \infty ; L^{2}\left(\mathbb{R}_{+}\right)\right), \quad\left(u_{x}-\tilde{u}_{x}, \theta_{x}-\tilde{\theta}_{x}\right) \in L^{2}\left(0, \infty ; H^{1}\left(\mathbb{R}_{+}\right)\right),
\end{gathered}
$$

and

$$
\lim _{t \rightarrow \infty} \sup _{x \in \mathbb{R}_{+}}|(\rho-\tilde{\rho}, u-\tilde{u}, \theta-\tilde{\theta})(t, x)|=0 .
$$

Remark 1.1. Theorem 1 shows that the non-degenerate stationary solution (i.e. the one which decays exponentially as (1.9)) is asymptotically stable even for large initial perturbation with general adiabatic exponent $\gamma$, provided that the boundary strength is small.

To derive the large-time behavior of solutions toward stationary solutions, it is sufficient to deduce certain uniform (with respect to the time $t$ ) a priori estimates on the perturbations. The essential step is to get the lower and upper positive bounds for both the density $\rho$ and the temperature $\theta$ uniformly in time and space. In case of small initial perturbation, as in [8], we can use the smallness of the a priori $H^{1}$-norm of the perturbation to obtain the uniform bounds of the density $\rho$ and the temperature $\theta$. Based on such uniform bounds and the smallness of the boundary strength $\delta$, one can derive certain uniform a priori $H^{1}$-norm energy type estimates on the perturbation. In case that the adiabatic exponent $\gamma$ is close to 1 , as in [22], we can control the lower and upper bounds of $\theta$, and by using the Kanel's technique, we can then obtain the uniform positive bounds for $\rho$ provided the boundary strength $\delta$ is small.

We are interested in showing the stability of the non-degenerate stationary solution under large initial perturbation with general $\gamma$. For this purpose, we first deduce the basic energy estimate with the aid 
of the decay property of the non-degenerate stationary solution provided that the boundary strength $\delta$ multiplied with a certain function of the a priori lower and upper bounds of density $\rho$ and temperature $\theta$ is suitably small (see Lemma 2.2). Next, to get uniform pointwise bounds of the density $\rho$, we transform the outflow problem (1.1)-(1.5) into a free boundary problem in the Lagrangian coordinate. By modifying Jiang's argument in $[6,7]$ for fixed domains, we will use a cut-off function with parameter to localize the free boundary problem, and then we will deduce a local representation of the specific volume $v=1 / \rho$ to establish the uniform bounds of $v$. With such uniform bounds of the density $\rho$ in hand, we can derive the $H^{1}$-norm (in the spatial variable $x$ ) estimate of the perturbation $\theta(t, x)-\tilde{\theta}(x)$ uniformly in the time $t$ in the Eulerian coordinate. Then the uniform upper bound of the temperature $\theta$ is obtained in light of the Cauchy's inequality. We note that our derivation of the uniform upper bound of $\theta$ is motivated by the recent work [14], which is concerned with the stability of the constant state to the compressible Navier-Stokes equations on unbounded but fixed domains in the Lagrangian coordinate. The maximum principle gives us the lower bound of the temperature $\theta$ locally in time $t$. In view of the a priori assumption (2.6), we have to get the uniform positive lower bound of the temperature $\theta$, which will be achieved by combining the local lower bound of $\theta$ and the detailed continuation argument.

Another interesting problem is on the asymptotical stability of the rarefaction wave and its superposition with the non-degenerate stationary solution to the outflow problem (1.1)-(1.5) under large initial perturbation. See $[5,10,11]$ for the isentropic case and [22] for the case of small initial perturbation. We will consider this problem in a forthcoming paper.

The layout of this paper is as follows. After stating the notations, in section 2, we establish the desired energy estimates and obtain the uniform bounds of both density and temperature. In section 3 , we extend the local solution step by step to a global one and prove the stability of the stationary solution by combining the a priori estimates obtained in section 2 with the the continuation argument.

Notation. We employ $C$ or $C_{i}(i \in \mathbb{N})$ to denote a generic positive constant which is independent of $t$, $x$ and $\delta$. Notice that all the constants may change from line to line. The Gaussian bracket $[x]$ means the largest integer not greater than $x$. For function spaces, $L^{p}\left(\mathbb{R}_{+}\right)(1 \leq p \leq \infty)$ stands for the usual Lebesgue space on $\mathbb{R}_{+}$equipped with the norm $\|\cdot\|_{L^{p}}$ and $H^{k}\left(\mathbb{R}_{+}\right)$the usual Sobolev space in the $L^{2}$ sense with norm $\|\cdot\|_{k}$. We use the notation $\|\cdot\|=\|\cdot\|_{L^{2}}$. We denote by $C^{k}\left(I ; H^{p}\right)$ the space of $k$-times continuously differentiable functions on the interval $I$ with values in $H^{p}\left(\mathbb{R}_{+}\right)$and $L^{2}\left(I ; H^{p}\right)$ the space of $L^{2}$-functions on $I$ with values in $H^{p}\left(\mathbb{R}_{+}\right)$.

\section{A priori estimates}

\subsection{Reformation of the problem}

This section is devoted to deriving a priori estimates on the solution $(\rho, u, \theta)$ to the outflow problem (1.1)-(1.5). To this end, we regard the solution $(\rho, u, \theta)$ as a perturbation from the stationary solution $(\tilde{\rho}, \tilde{u}, \tilde{\theta})$ and put the perturbation $(\phi, \psi, \vartheta)$ by

$$
(\phi, \psi, \vartheta)(t, x):=(\rho, u, \theta)(t, x)-(\tilde{\rho}, \tilde{u}, \tilde{\theta})(x) .
$$

Subtracting (1.6)-(1.7) from (1.1)-(1.5) yields

$$
\left\{\begin{aligned}
\phi_{t}+u \phi_{x}+\rho \psi_{x} & =-\tilde{u}_{x} \phi-\tilde{\rho}_{x} \psi, \quad t>0, x \in \mathbb{R}_{+} \\
\rho\left(\psi_{t}+u \psi_{x}\right)+(P-\tilde{P})_{x} & =\mu \psi_{x x}+g \\
c_{v} \rho\left(\vartheta_{t}+u \vartheta_{x}\right)+P \psi_{x} & =\kappa \vartheta_{x x}+\mu \psi_{x}^{2}+h \\
\left.(\phi, \psi, \vartheta)\right|_{t=0} & =\left(\phi_{0}, \psi_{0}, \vartheta_{0}\right) \\
\left.(\psi, \vartheta)\right|_{x=0} & =(0,0)
\end{aligned}\right.
$$

where

$$
g:=-\tilde{u}_{x}(\tilde{u} \phi+\rho \psi), \quad h:=-c_{v} \tilde{\theta}_{x}(\tilde{u} \phi+\rho \psi)-\tilde{u}_{x}(P-\tilde{P})+2 \mu \tilde{u}_{x} \psi_{x}
$$


and the initial condition $\left(\phi_{0}, \psi_{0}, \vartheta_{0}\right):=\left(\rho_{0}-\tilde{\rho}, u_{0}-\tilde{u}, \theta_{0}-\tilde{\theta}\right)$ satisfies

$$
\left(\phi_{0}, \psi_{0}, \vartheta_{0}\right)(x) \rightarrow(0,0,0), \quad \text { as } x \rightarrow \infty .
$$

The solution space $X\left(0, T ; m_{1}, M_{1} ; m_{2}, M_{2}\right)$ is defined by

$$
\begin{aligned}
X\left(0, T ; m_{1}, M_{1} ; m_{2}, M_{2}\right):=\left\{(\phi, \psi, \vartheta) \in C\left([0, T] ; H^{1}\right):\left(\psi_{x}, \vartheta_{x}\right) \in L^{2}\left(0, T ; H^{1}\right),\right. & \\
& \left.\phi_{x} \in L^{2}\left(0, T ; L^{2}\right), m_{1} \leq \phi+\tilde{\rho} \leq M_{1}, m_{2} \leq \vartheta+\tilde{\theta} \leq M_{2}\right\} .
\end{aligned}
$$

We summarize the local existence of solutions to the problem (2.2) in the following proposition, which can be proved by the standard iteration method (see [4]).

Proposition 2.1 (Local existence). Suppose that the conditions in Theorem 1 hold. If $\left\|\left(\phi_{0}, \psi_{0}, \vartheta_{0}\right)\right\|_{1} \leq$ $M, \lambda_{1} \leq \phi_{0}(x)+\tilde{\rho}(x) \leq \Lambda_{1}$, and $\lambda_{2} \leq \vartheta_{0}(x)+\tilde{\theta}(x) \leq \Lambda_{2}$ hold for all $x \in \mathbb{R}_{+}$, then (2.2) admits a unique solution $(\phi, \psi, \vartheta) \in X\left(0, T_{0} ; \frac{1}{2} \lambda_{1}, 2 \Lambda_{1} ; \frac{1}{2} \lambda_{2}, 2 \Lambda_{2}\right)$ for some constant $T_{0}=T_{0}\left(\lambda_{1}, \lambda_{2}, M\right)>0$ depending only on $\lambda_{1}, \lambda_{2}$ and $M$.

We now turn to deduce certain uniform a priori estimates for the perturbation $(\phi, \psi, \vartheta) \in X\left(0, T ; m_{1}, M_{1}, m_{2}, M_{2}\right)$ in the Sobolev space $H^{1}$. Before doing so, we recall that $C$ or $C_{i}(i \in \mathbb{N})$ will be used to denote some generic positive constant which depends only on $\inf _{\mathbb{R}_{+}}\left(\rho_{0}, \theta_{0}\right)$ and $\left\|\left(\phi_{0}, \psi_{0}, \vartheta_{0}\right)\right\|_{1}$. For notational simplicity, we introduce $A \lesssim B$ if $A \leq C B$ holds uniformly for some constant $C$ depending only on $\inf _{\mathbb{R}_{+}}\left(\rho_{0}, \theta_{0}\right)$ and $\left\|\left(\phi_{0}, \psi_{0}, \vartheta_{0}\right)\right\|_{1}$. The notation $A \sim B$ means that both $A \lesssim B$ and $B \lesssim A$. Besides, we will use the notation $(\rho, \theta)=(\phi+\tilde{\rho}, \vartheta+\tilde{\theta})$ so that

$$
m_{1} \leq \rho(t, x) \leq M_{1}, \quad m_{2} \leq \theta(t, x) \leq M_{2}, \quad \text { for all }(t, x) \in[0, T] \times \mathbb{R}_{+} .
$$

Without loss of generality, we may assume that $m_{i} \leq 1 \leq M_{i}$ for $i=1,2$.

\subsection{Basic energy estimate}

First, we have the following basic energy estimate.

Lemma 2.2. There exists a sufficiently small $\epsilon_{0}>0$ such that if

$$
\Xi\left(m_{1}, M_{1}, m_{2}, M_{2}\right) \delta \leq \epsilon_{0}, \quad \Xi\left(m_{1}, M_{1}, m_{2}, M_{2}\right):=m_{1}^{-10} M_{1}^{10} m_{2}^{-10} M_{2}^{10},
$$

then

$$
\begin{aligned}
& \int_{\mathbb{R}_{+}} \frac{\phi_{x}^{2}}{\rho^{3}}+\int_{0}^{t} \frac{\phi_{x}^{2}}{\rho^{3}}(s, 0) d s+\int_{0}^{t} \int_{\mathbb{R}_{+}} \frac{\theta \phi_{x}^{2}}{\rho^{2}} \lesssim 1+\|\theta\|_{L^{\infty}\left([0, T] \times \mathbb{R}_{+}\right)}, \\
& \int_{\mathbb{R}_{+}} \rho \mathcal{E}+\int_{0}^{t} \rho \Phi\left(\frac{\tilde{\rho}}{\rho}\right)(s, 0) d s+\int_{0}^{t} \int_{\mathbb{R}_{+}}\left[\frac{\psi_{x}^{2}}{\theta}+\frac{\vartheta_{x}^{2}}{\theta^{2}}\right] \lesssim 1
\end{aligned}
$$

where

$$
\mathcal{E}:=R \tilde{\theta} \Phi\left(\frac{\tilde{\rho}}{\rho}\right)+\frac{1}{2} \psi^{2}+c_{v} \tilde{\theta} \Phi\left(\frac{\theta}{\tilde{\theta}}\right), \quad \Phi(z):=z-\ln z-1 .
$$

Proof. By a straightforward calculation, we find

$$
(\rho \mathcal{E})_{t}+\left[\rho u \mathcal{E}+\psi(P-\tilde{P})-\mu \psi \psi_{x}-\kappa \frac{\vartheta \vartheta_{x}}{\theta}\right]_{x}+\mu \frac{\tilde{\theta} \psi_{x}^{2}}{\theta}+\kappa \frac{\tilde{\theta} \vartheta_{x}^{2}}{\theta^{2}}=\mathcal{R},
$$

where

$$
\begin{aligned}
\mathcal{R}= & \rho \tilde{\theta}_{x}\left[R \tilde{u} \Phi\left(\frac{\tilde{\rho}}{\rho}\right)+c_{v} \tilde{u} \Phi\left(\frac{\theta}{\tilde{\theta}}\right)-c_{v} \psi \ln \left(\frac{\theta}{\tilde{\theta}}\right)-R \psi \ln \left(\frac{\tilde{\rho}}{\rho}\right)\right]-\rho \tilde{u}_{x} \psi^{2} \\
& -\left(c_{v} \tilde{\rho} \tilde{u} \tilde{\theta}_{x}+\tilde{P} \tilde{u}_{x}\right)\left(\frac{\phi \vartheta}{\tilde{\rho} \tilde{\theta}}+\frac{\vartheta^{2}}{\tilde{\theta} \theta}\right)+2 \mu \frac{\tilde{u}_{x} \psi_{x} \vartheta}{\theta}+\kappa \frac{\tilde{\theta}_{x} \vartheta \vartheta_{x}}{\theta^{2}}-\mu \frac{\tilde{u}_{x x} \phi \psi}{\tilde{\rho}} .
\end{aligned}
$$


The identities

$$
\Phi(z)=\int_{0}^{1} \int_{0}^{1} \theta_{1} \Phi^{\prime \prime}\left(1+\theta_{1} \theta_{2}(z-1)\right) d \theta_{2} d \theta_{1}(z-1)^{2}
$$

and

$$
\ln z=\int_{0}^{1} \frac{(z-1) d \theta_{1}}{1+\theta_{1}(z-1)}
$$

imply

$$
\Phi(z)+|\ln z|^{2} \lesssim\left(z^{-1}+1\right)^{2}(z-1)^{2}, \quad(z+1)^{-2}(z-1)^{2} \lesssim \Phi(z) .
$$

In view of (2.5) and (2.11), we apply Cauchy's inequality to $\mathcal{R}$ and obtain

$$
\mathcal{R} \lesssim\left|\left(\tilde{\rho}_{x}, \tilde{u}_{x}, \tilde{\theta}_{x}, \tilde{u}_{x x}\right)\right|\left[M_{1} m_{1}^{-2} \phi^{2}+M_{1} m_{2}^{-2} \vartheta^{2}+M_{1} \psi^{2}+\frac{\psi_{x}^{2}}{\theta}+\frac{\vartheta_{x}^{2}}{\theta^{2}}\right]
$$

Integrate $(2.10)$ over $[0, T] \times \mathbb{R}_{+}$and use the good sign of $u_{-}$to derive

$$
\begin{aligned}
& \int_{\mathbb{R}_{+}} \rho \mathcal{E}+\int_{0}^{t} \rho \Phi\left(\frac{\tilde{\rho}}{\rho}\right)(s, 0) d s+\int_{0}^{t} \int_{\mathbb{R}_{+}}\left[\frac{\psi_{x}^{2}}{\theta}+\frac{\vartheta_{x}^{2}}{\theta^{2}}\right] \\
& \lesssim 1+\int_{0}^{t} \int_{\mathbb{R}_{+}}\left|\left(\tilde{\rho}_{x}, \tilde{u}_{x}, \tilde{\theta}_{x}, \tilde{u}_{x x}\right)\right|\left[M_{1} m_{1}^{-2} \phi^{2}+M_{1} m_{2}^{-2} \vartheta^{2}+M_{1} \psi^{2}+\frac{\psi_{x}^{2}}{\theta}+\frac{\vartheta_{x}^{2}}{\theta^{2}}\right] .
\end{aligned}
$$

To estimate the terms on the right-hand side of (2.12), we use an idea in Nikkuni-Kawashima [20], i.e. a Poincaré type inequality

$$
|\varphi(t, x)| \leq|\varphi(t, 0)|+\sqrt{x}\left\|\varphi_{x}(t)\right\|, \quad x \in \mathbb{R}_{+}
$$

Applying this inequality to $\phi, \psi$ and $\vartheta$, we deduce from (1.9) and (2.11) that

$$
\begin{aligned}
\sum_{l=0,1} \int_{0}^{t} \int_{\mathbb{R}_{+}}\left|\partial_{x}^{k}(\tilde{\rho}, \tilde{u}, \tilde{\theta})\right|\left|\partial_{x}^{l}(\phi, \psi, \vartheta)\right|^{2} \\
\quad \lesssim \delta \int_{0}^{t}\left[|\phi(s, 0)|^{2}+\left\|\left(\phi_{x}, \psi_{x}, \vartheta_{x}\right)(s)\right\|^{2}\right] d s \\
\quad \lesssim M_{1}^{2} m_{1}^{-1} \delta \int_{0}^{t} \rho \Phi\left(\frac{\tilde{\rho}}{\rho}\right)(s, 0) d s \\
\quad+\delta \int_{0}^{t}\left\|\phi_{x}(s)\right\|^{2} d s+M_{2}^{2} \delta \int_{0}^{t} \int_{\mathbb{R}_{+}}\left[\frac{\psi_{x}^{2}}{\theta}+\frac{\vartheta_{x}^{2}}{\theta^{2}}\right]
\end{aligned}
$$

for each integer $k=1,2, \cdots$. Plug (2.13) into (2.12) to derive

$$
\begin{aligned}
& \int_{\mathbb{R}_{+}} \rho \mathcal{E}+\int_{0}^{t} \rho \Phi\left(\frac{\tilde{\rho}}{\rho}\right)(s, 0) d s+\int_{0}^{t} \int_{\mathbb{R}_{+}}\left[\frac{\psi_{x}^{2}}{\theta}+\frac{\vartheta_{x}^{2}}{\theta^{2}}\right] \\
& \lesssim 1+m_{1}^{-3} M_{1}^{3} m_{2}^{-2} \delta \int_{0}^{t} \rho \Phi\left(\frac{\tilde{\rho}}{\rho}\right)(s, 0) d s \\
& \quad+m_{1}^{-2} M_{1} m_{2}^{-2} \delta \int_{0}^{t}\left\|\phi_{x}(s)\right\|^{2} d s+m_{1}^{-2} M_{1} m_{2}^{-2} M_{2}^{2} \int_{0}^{t} \int_{\mathbb{R}_{+}}\left[\frac{\psi_{x}^{2}}{\theta}+\frac{\vartheta_{x}^{2}}{\theta^{2}}\right]
\end{aligned}
$$

which implies

$$
\begin{aligned}
& \int_{\mathbb{R}_{+}} \rho \mathcal{E}+\int_{0}^{t} \rho \Phi\left(\frac{\tilde{\rho}}{\rho}\right)(s, 0) d s+\int_{0}^{t} \int_{\mathbb{R}_{+}}\left[\frac{\psi_{x}^{2}}{\theta}+\frac{\vartheta_{x}^{2}}{\theta^{2}}\right] \\
& \lesssim 1+m_{1}^{-2} M_{1} m_{2}^{-2} \delta \int_{0}^{t}\left\|\phi_{x}(s)\right\|^{2} d s
\end{aligned}
$$


provided (2.6) holds for a suitable small $\epsilon_{0}>0$.

To control the last term of (2.14), we differentiate $(2.2)_{1}$ (first equation of (2.2)) with respect to $x$, and then multiply the resulting identity and $(2.2)_{2}$ by $\frac{\phi_{x}}{\rho^{3}}$ and $\frac{\phi_{x}}{\mu \rho^{2}}$, respectively, to discover

$$
\left[\frac{\phi_{x}^{2}}{2 \rho^{3}}+\frac{\phi_{x} \psi}{\mu \rho}\right]_{t}+\left[\frac{u \phi_{x}^{2}}{2 \rho^{3}}-\frac{\psi \phi_{t}}{\mu \rho}-\frac{\tilde{\rho}_{x} \psi^{2}}{\mu \rho}\right]_{x}+\frac{R \theta \phi_{x}^{2}}{\mu \rho^{2}}=Q
$$

with

$$
\begin{aligned}
Q= & \frac{\psi_{x}^{2}}{\mu}-\frac{R \phi_{x} \vartheta_{x}}{\mu \rho}-\left[\frac{\tilde{u} \tilde{u}_{x}}{\mu \rho^{2}}+\frac{R \tilde{\theta}_{x}}{\mu \rho^{2}}+\frac{\tilde{u}_{x x}}{\rho^{3}}\right] \phi \phi_{x}-\left[\frac{2 \tilde{\rho}_{x} \psi_{x}}{\rho^{3}}+\frac{R \tilde{\rho}_{x} \vartheta}{\mu \rho^{2}}\right] \phi_{x} \\
& -\left[\frac{2 \tilde{\rho}_{x} \psi}{\mu \rho}-\frac{\tilde{u}_{x} \phi}{\mu \rho}\right] \psi_{x}-\left[\frac{\tilde{u}_{x}}{\mu \rho}-\frac{\tilde{\rho} \tilde{u}_{x}}{\mu \rho^{2}}+\frac{\tilde{\rho}_{x x}}{\rho^{3}}\right] \psi \phi_{x}-\frac{\tilde{\rho}_{x} \tilde{u}_{x} \phi \psi}{\mu \rho^{2}}-\frac{\tilde{\rho}_{x x} \psi^{2}}{\mu \rho} .
\end{aligned}
$$

We integrate (2.15) over $[0, t] \times \mathbb{R}_{+}$and use Cauchy's inequality to have

$$
\int_{\mathbb{R}_{+}} \frac{\phi_{x}^{2}}{\rho^{3}}+\int_{0}^{t} \frac{\phi_{x}^{2}}{\rho^{3}}(s, 0)+\int_{0}^{t} \int_{\mathbb{R}_{+}} \frac{\theta \phi_{x}^{2}}{\rho^{2}} \lesssim 1+\int_{\mathbb{R}_{+}} \rho \psi^{2}+\int_{0}^{t} \int_{\mathbb{R}_{+}}|Q| .
$$

Cauchy's inequality yields the bound

$$
|Q| \lesssim \psi_{x}^{2}+C(\epsilon) \frac{\vartheta_{x}^{2}}{\theta}+\epsilon \frac{\theta \phi_{x}^{2}}{\rho^{2}}+m_{1}^{-3}\left|\left(\tilde{\rho}_{x}, \tilde{u}_{x}, \tilde{\theta}_{x}, \tilde{\rho}_{x x}, \tilde{u}_{x x}\right)\right|\left|\left(\phi, \psi, \vartheta, \phi_{x}, \psi_{x}\right)\right|^{2} .
$$

Plugging this inequality into (2.16), we take $\epsilon>0$ suitable small and utilize (2.13) and (2.14) to obtain

$$
\begin{aligned}
& \int_{\mathbb{R}_{+}} \frac{\phi_{x}^{2}}{\rho^{3}}+\int_{0}^{t} \frac{\phi_{x}^{2}}{\rho^{3}}(s, 0)+\int_{0}^{t} \int_{\mathbb{R}_{+}} \frac{\theta \phi_{x}^{2}}{\rho^{2}} \\
& \lesssim 1+\int_{0}^{t} \int_{\mathbb{R}_{+}}\left[\psi_{x}^{2}+\frac{\vartheta_{x}^{2}}{\theta}\right]+m_{1}^{-3} M_{1}^{3} m_{2}^{-2} M_{2}^{2} \delta \int_{0}^{t}\left\|\phi_{x}(s)\right\|^{2} d s \\
& \quad+m_{1}^{-4} M_{1}^{2} M_{2}^{2} \delta\left[\int_{0}^{t} \rho \Phi\left(\frac{\tilde{\rho}}{\rho}\right)(s, 0) d s+\int_{0}^{t} \int_{\mathbb{R}_{+}}\left(\frac{\psi_{x}^{2}}{\theta}+\frac{\vartheta_{x}^{2}}{\theta^{2}}\right)\right] \\
& \lesssim 1+\int_{0}^{t} \int_{\mathbb{R}_{+}}\left[\psi_{x}^{2}+\frac{\vartheta_{x}^{2}}{\theta}\right]+m_{1}^{-10} M_{1}^{10} m_{2}^{-10} M_{2}^{10} \delta \int_{0}^{t} \int_{\mathbb{R}_{+}} \frac{\theta \phi_{x}^{2}}{\rho^{2}}
\end{aligned}
$$

Taking $\epsilon_{0}>0$ small enough, we can derive from (2.6) that

$$
\int_{\mathbb{R}_{+}} \frac{\phi_{x}^{2}}{\rho^{3}}+\int_{0}^{t} \frac{\phi_{x}^{2}}{\rho^{3}}(s, 0)+\int_{0}^{t} \int_{\mathbb{R}_{+}} \frac{\theta \phi_{x}^{2}}{\rho^{2}} \lesssim 1+\int_{0}^{t} \int_{\mathbb{R}_{+}}\left[\psi_{x}^{2}+\frac{\vartheta_{x}^{2}}{\theta}\right] .
$$

The estimate (2.7) follows by plugging (2.14) into (2.17) under the condition (2.6) for a sufficiently small $\epsilon_{0}>0$. We plug (2.7) into (2.14) to deduce the estimate (2.8). The proof of the lemma is completed.

\subsection{Pointwise estimates of density}

Once the basic energy estimate (2.8) is obtained, we can proceed to deduce the positive lower and upper bounds of the density uniformly in time. For this purpose, it is easier to consider the problem in the Lagrangian coordinate than in the Eulerian coordinate. We introduce the Lagrangian variable

$$
y=-u_{-} \int_{0}^{t} \rho(s, 0) d s+\int_{0}^{x} \rho(t, z) d z
$$

and define $(\hat{\rho}, \hat{u}, \hat{\theta})(t, y):=(\rho, u, \theta)(t, x)$. By the coordinate change $(t, x) \mapsto(t, y)$, the domain $[0, T] \times \mathbb{R}_{+}$ is mapped into

$$
\Omega_{T}:=\{(t, y): 0 \leq t \leq T, y>Y(t)\},
$$


where

$$
Y(t):=-u_{-} \int_{0}^{t} \rho(s, 0) d s .
$$

The outflow problem (1.1)-(1.5) can be transformed into the problem in the Lagrangian coordinate:

$$
\left\{\begin{array}{rlrl}
v_{t}-u_{y} & =0 & & \\
u_{t}+P_{y} & =\left(\frac{\mu u_{y}}{v}\right)_{y} & & \\
\left(c_{v} \theta+\frac{u^{2}}{2}\right)_{t}+(P u)_{y} & =\left(\frac{\kappa \theta_{y}}{v}+\frac{\mu u u_{y}}{v}\right)_{y} \\
\left.(u, \theta)\right|_{y=Y(t)} & =\left(u_{-}, \theta_{-}\right) \\
\left.(v, u, \theta)\right|_{t=0} & =\left(v_{0}, u_{0}, \theta_{0}\right)
\end{array}\right.
$$

where $v=1 / \rho$ stands for the specific volume of the gas. We drop the hats in the formula in this subsection for simplicity of notation. The basic energy estimate (2.8) in Eulerian coordinate can be transformed into a corresponding estimate in Lagrangian coordinate as a corollary of Lemma 2.2.

Corollary 2.3. If (2.6) holds for a sufficiently small $\epsilon_{0}>0$, then

$$
\sup _{0 \leq t \leq T} \int_{Y(t)}^{\infty} \mathcal{E} d y+\int_{0}^{T} \int_{Y(t)}^{\infty}\left[\frac{\psi_{y}^{2}}{v \theta}+\frac{\vartheta_{y}^{2}}{v \theta^{2}}\right] \lesssim 1
$$

Note that the function $Y(t)$ describing the boundary in the Lagrangian coordinate is part of the unknown, i.e. the problem (2.19) is a free boundary problem. To obtain the uniform bounds of the specific volume $v$ for the free boundary problem (2.19), we introduce

$$
\Omega_{i}(t):= \begin{cases}{[Y(t),[Y(t)]+2],} & i=[Y(t)]+1 \\ {[i, i+1],} & i \geq[Y(t)]+2\end{cases}
$$

for any integer $i \geq[Y(t)]+1$. Based on the basic energy estimate (2.20), we have the following lemma.

Lemma 2.4. Suppose that (2.6) holds for a sufficiently small $\epsilon_{0}>0$. Then there exists a constant $C_{0}>0$, which depends only on $\inf _{\mathbb{R}_{+}}\left(\rho_{0}, \theta_{0}\right)$ and $\left\|\left(\phi_{0}, \psi_{0}, \vartheta_{0}\right)\right\|_{1}$, such that for all pair $(s, t)$ with $0 \leq s \leq t \leq T$ and integer $i \geq[Y(t)]+1$,

$$
C_{0}^{-1} \leq \int_{\Omega_{i}(t)} v(s, y) d y, \int_{\Omega_{i}(t)} \theta(s, y) d y \leq C_{0},
$$

and there are points $a_{i}(s, t), b_{i}(s, t) \in \Omega_{i}(t)$ such that

$$
C_{0}^{-1} \leq v\left(s, a_{i}(s, t)\right), \theta\left(s, b_{i}(s, t)\right) \leq C_{0}
$$

Proof. Let $0 \leq s \leq t \leq T$ and $i \geq[Y(t)]+1$. From the definition of $Y(t)$ and the sign of $u_{-}$, we have $Y(s) \leq Y(t)$ and $\Omega_{i}(t) \subset[Y(s), \infty)$. In view of $(2.20)$, we get

$$
\int_{\Omega_{i}(t)} \Phi\left(\frac{v}{\tilde{v}}\right)(s, y) d y+\int_{\Omega_{i}(t)} \Phi\left(\frac{\theta}{\tilde{\theta}}\right)(s, y) d y \lesssim 1 .
$$

Apply Jensen's inequality to the convex function $\Phi$ to obtain

$$
\Phi\left(\frac{1}{\left|\Omega_{i}(t)\right|} \int_{\Omega_{i}(t)} \frac{v}{\tilde{v}}(s, y) d y\right)+\Phi\left(\frac{1}{\left|\Omega_{i}(t)\right|} \int_{\Omega_{i}(t)} \frac{\theta}{\tilde{\theta}}(s, y) d y\right) \leq C
$$


Let $\alpha$ and $\beta$ be the two positive roots of the equation $\Phi(z)=C$. Then we have

$$
\alpha \leq \frac{1}{\left|\Omega_{i}(t)\right|} \int_{\Omega_{i}(t)} \frac{v}{\tilde{v}}(s, y) d y, \frac{1}{\left|\Omega_{i}(t)\right|} \int_{\Omega_{i}(t)} \frac{\theta}{\tilde{\theta}}(s, y) d y \leq \beta .
$$

These estimates imply (2.22). Finally we employ the mean value theorem to $(2.22)$ to find $a_{i}(s, t), b_{i}(s, t) \in$ $\Omega_{i}(t)$ satisfying (2.23). The proof of the lemma is completed.

We deduce a local representation of $v$ in the next lemma by modifying Jiang's argument in $[6,7]$ for fixed domains. To this end, we introduce the cutoff function $\varphi_{z} \in W^{1, \infty}(\mathbb{R})$ with parameter $z \in \mathbb{R}$ by

$$
\varphi_{z}(y)= \begin{cases}1, & y<[z]+4, \\ {[z]+5-y,} & {[z]+4 \leq y<[z]+5,} \\ 0, & y \geq[z]+5 .\end{cases}
$$

Lemma 2.5. Let $(\tau, z) \in \Omega_{T}$. Then we have

$$
v(t, y)=B_{z}(t, y) A_{z}(t)+\frac{R}{\mu} \int_{0}^{t} \frac{B_{z}(t, y) A_{z}(t)}{B_{z}(s, y) A_{z}(s)} \theta(s, y) d s
$$

for all $t \in[0, \tau]$ and $y \in I_{z}(\tau):=(Y(\tau), \infty) \cap([z]-1,[z]+4)$, where

$$
\begin{aligned}
B_{z}(t, y) & :=v_{0}(y) \exp \left\{\frac{1}{\mu} \int_{y}^{\infty}\left(u_{0}(\xi)-u(t, \xi)\right) \varphi_{z}(\xi) d \xi\right\}, \\
A_{z}(t) & :=\exp \left\{\frac{1}{\mu} \int_{0}^{t} \int_{[z]+4}^{[z]+5}\left(\frac{\mu u_{y}}{v}-P\right) d \xi d s\right\} .
\end{aligned}
$$

Proof. We multiply $(2.19)_{2}$ by $\varphi_{z}$ to get

$$
\left(\varphi_{z} u\right)_{t}=\left[\left(\mu \frac{u_{y}}{v}-P\right) \varphi_{z}\right]_{y}-\varphi_{z}^{\prime}\left(\mu \frac{u_{y}}{v}-P\right) .
$$

Let $(t, y) \in[0, \tau] \times I_{z}(\tau)$. Since $y>Y(s)$ for each $s \in[0, \tau]$, we have $[0, \tau] \times[y, \infty) \subset \Omega_{T}$. In view of the identity $\varphi_{z}(y)=1$ and $(2.19)_{1}$, we integrate $(2.28)$ over $[0, t] \times[y, \infty)$ to get

$$
-\int_{y}^{\infty} \varphi_{z}(\xi)\left(u(t, \xi)-u_{0}(\xi)\right) d \xi=\mu \ln \frac{v(t, y)}{v_{0}(y)}-R \int_{0}^{t} \frac{\theta(s, y)}{v(s, y)} d s+\int_{0}^{t} \int_{[z]+4}^{[z]+5}\left(P-\mu \frac{u_{y}}{v}\right) .
$$

This implies that for each $t \in[0, \tau]$,

$$
\frac{1}{v(t, y)} \exp \left\{\frac{R}{\mu} \int_{0}^{t} \frac{\theta(s, y)}{v(s, y)} d s\right\}=\frac{1}{B_{z}(t, y) A_{z}(t)} .
$$

Multiplying (2.29) by $R \theta(t, y) / \mu$ and integrating the resulting identity over $[0, t]$, we have

$$
\exp \left\{\frac{R}{\mu} \int_{0}^{t} \frac{\theta(s, y)}{v(s, y)} d s\right\}=1+\frac{R}{\mu} \int_{0}^{t} \frac{\theta(s, y)}{B_{z}(s, y) A_{z}(s)} d s .
$$

We then plug this identity into (2.29) to obtain (2.25) and complete the proof of the lemma.

The following lemma is devoted to showing the bounds of the specific volume $v(\tau, z)$ uniformly in the time $\tau$ and the Lagrangian variable $z$.

Lemma 2.6. If (2.6) holds for a sufficiently small $\epsilon_{0}>0$, then

$$
C_{1}^{-1} \leq v(\tau, z) \leq C_{1} \quad \text { for all }(\tau, z) \in \Omega_{T} .
$$


Proof. Let $(\tau, z) \in \Omega_{T}$ be arbitrary but fixed. The proof is divided into three steps.

Step 1. In view of Cauchy's inequality and (2.20), we have

$$
B_{z}(t, y) \sim 1 \quad \text { for all }(t, y) \in[0, \tau] \times I_{z}(\tau) .
$$

Let $0 \leq s \leq t \leq \tau$. Apply Cauchy's inequality and Jensen's inequality for the convex function $1 / x$ $(x>0),(1.9),(2.20)$ and $(2.22)$ to deduce

$$
\begin{aligned}
& \int_{s}^{t} \int_{[z]+4}^{[z]+5}\left[\frac{\mu u_{y}}{v}-P\right] \\
& \leq C \int_{s}^{t} \int_{[z]+4}^{[z]+5} \frac{u_{y}^{2}}{v \theta}+\frac{R}{2} \int_{s}^{t} \int_{[z]+4}^{[z]+5} \frac{\theta}{v}-\int_{s}^{t} \int_{[z]+4}^{[z]+5} \frac{R \theta}{v} \\
& \leq C \int_{s}^{t} \int_{[z]+4}^{[z]+5}\left[\frac{\psi_{y}^{2}}{v \theta}+\frac{\tilde{u}_{y}^{2}}{v \theta}\right]-\frac{R}{2} \int_{s}^{t} \int_{[z]+4}^{[z]+5} \frac{\theta}{v} \\
& \leq C+C M_{1} m_{2}^{-1} \delta(t-s)-\frac{R}{2} \int_{s}^{t} \inf _{([z]+4,[z]+5)} \theta\left(t^{\prime}, \cdot\right)\left[\int_{[z]+4}^{[z]+5} v\right]^{-1} d t^{\prime} \\
& \leq C+C \epsilon_{0}(t-s)-C^{-1} \int_{s}^{t} \inf _{([z]+4,[z]+5)} \theta\left(t^{\prime}, \cdot\right) d t^{\prime},
\end{aligned}
$$

For each $0 \leq t^{\prime} \leq t$, there exists $y\left(t^{\prime}\right) \in[[z]+4,[z]+5]$ such that

$$
\theta\left(t^{\prime}, y\left(t^{\prime}\right)\right)=\inf _{([z]+4,[z]+5)} \theta\left(t^{\prime}, \cdot\right),
$$

Since $[z]+4 \geq\left[Y\left(t^{\prime}\right)\right]+2$, we derive from the definition $(2.21)$ that $\Omega_{[z]+4}\left(t^{\prime}\right)=[[z]+4,[z]+5]$. We then apply Hölder's and Cauchy's inequalities to obtain from (1.9), Lemma 2.4 and (2.20) that

$$
\begin{aligned}
& \left|\int_{s}^{t} \int_{b_{[z]+4}\left(t^{\prime}, t^{\prime}\right)}^{y\left(t^{\prime}\right)} \frac{\theta_{y}}{\theta}\left(t^{\prime}, \xi\right) d \xi d t^{\prime}\right| \\
& \leq \int_{s}^{t} \int_{\Omega_{[z]+4}\left(t^{\prime}\right)}\left|\frac{\tilde{\theta}_{y}}{\theta}\left(t^{\prime}, \xi\right)+\frac{\vartheta_{y}}{\theta}\left(t^{\prime}, \xi\right)\right| d \xi d t^{\prime} \\
& \leq m_{2}^{-1} \delta(t-s)+\int_{s}^{t}\left|\int_{Y\left(t^{\prime}\right)}^{\infty} \frac{\vartheta_{y}^{2}}{v \theta^{2}}\left(t^{\prime}, \xi\right) d \xi\right|^{\frac{1}{2}}\left|\int_{\Omega_{[z]+4}\left(t^{\prime}\right)} v\left(t^{\prime}, \xi\right) d \xi\right|^{\frac{1}{2}} d t^{\prime} \\
& \leq C(t-s)+C \int_{s}^{t} \int_{Y\left(t^{\prime}\right)}^{\infty} \frac{\vartheta_{y}^{2}}{v \theta^{2}}\left(t^{\prime}, \xi\right) d \xi d t^{\prime} \\
& \leq C(t-s)+C .
\end{aligned}
$$

Applying Jensen's inequality to the convex function $e^{x}$, we have from (2.23) and (2.33) that

$$
\begin{aligned}
& \int_{s}^{t} \inf _{([z]+4,[z]+5)} \theta\left(t^{\prime}, \cdot\right) d t^{\prime}=\int_{s}^{t} \exp \left(\ln \theta\left(t^{\prime}, y\left(t^{\prime}\right)\right)\right) d t^{\prime} \\
& \geq(t-s) \exp \left(\frac{1}{t-s} \int_{s}^{t} \ln \theta\left(t^{\prime}, y\left(t^{\prime}\right)\right) d t^{\prime}\right) \\
& \geq(t-s) \exp \left(\frac{1}{t-s} \int_{s}^{t}\left[\int_{b_{[z]+4}\left(t^{\prime}, t^{\prime}\right)}^{y\left(t^{\prime}\right)} \frac{\theta_{y}}{\theta}\left(t^{\prime}, \xi\right) d \xi+\ln \theta\left(t^{\prime}, b_{[z]+4}\left(t^{\prime}, t^{\prime}\right)\right)\right] d t^{\prime}\right) \\
& \geq(t-s) \exp \left(-\ln C_{0}-\frac{1}{t-s}\left|\int_{s}^{t} \int_{b_{[z]+4}\left(t^{\prime}, t^{\prime}\right)}^{y\left(t^{\prime}\right)} \frac{\theta_{y}}{\theta}\left(t^{\prime}, \xi\right) d \xi d t^{\prime}\right|\right) \\
& \geq \frac{t-s}{C} \exp \left(-\frac{C}{t-s}\right) .
\end{aligned}
$$


This implies

$$
-\int_{s}^{t} \inf _{([z]+4,[z]+5)} \theta\left(t^{\prime}, \cdot\right) d t^{\prime} \leq \begin{cases}0, & \text { for } 0 \leq t-s \leq 1, \\ -C^{-1}(t-s) & \text { for } t-s \geq 1 .\end{cases}
$$

Plugging (2.34) into (2.32) and taking $\epsilon_{0}>0$ small enough, we have for each $s \in[0, t]$ that

$$
\int_{s}^{t} \int_{[z]+4}^{[z]+5}\left[\frac{\mu u_{y}}{v}-P\right] \leq C-C^{-1}(t-s) .
$$

According to the definition (2.27), we then obtain

$$
0 \leq A_{z}(t) \leq C \mathrm{e}^{-t / C}, \quad \frac{A_{z}(t)}{A_{z}(s)} \leq C \mathrm{e}^{-(t-s) / C} \quad \text { for all } 0 \leq s \leq t \leq \tau .
$$

Step 2. Plugging (2.31) and (2.35) into (2.25), we infer

$$
\int_{0}^{t} \frac{A_{z}(t)}{A_{z}(s)} \theta(s, y) d s \lesssim v(t, y) \lesssim 1+\int_{0}^{t} \theta(s, y) \mathrm{e}^{-\frac{t-s}{C}} d s
$$

for all $(t, y) \in[0, \tau] \times I_{z}(\tau)$. In light of the fundamental theorem of calculus, we infer from (1.9) and (2.22) that for $y \in I_{z}(\tau)$ and $0 \leq s \leq t \leq \tau$,

$$
\begin{aligned}
& \left|\theta(s, y)^{\frac{1}{2}}-\theta\left(s, b_{[z]+2}(s, \tau)\right)^{\frac{1}{2}}\right| \\
& \lesssim \int_{I_{z}(\tau)} \theta^{-\frac{1}{2}}\left|\theta_{y}\right|(s, \xi) d \xi \\
& \lesssim \int_{I_{z}(\tau)} \theta^{-\frac{1}{2}}\left|\tilde{\theta}_{y}\right|(s, \xi) d \xi+\int_{I_{z}(\tau)} \theta^{-\frac{1}{2}}\left|\vartheta_{y}\right|(s, \xi) d \xi \\
& \lesssim m_{2}^{-\frac{1}{2}} \delta+\left[\int_{I_{z}(\tau)} \frac{\vartheta_{y}^{2}}{v \theta^{2}}(s, \xi) d \xi\right]^{\frac{1}{2}}\left[\int_{I_{z}(\tau)} v \theta(s, \xi) d \xi\right]^{\frac{1}{2}} \\
& \lesssim m_{2}^{-\frac{1}{2}} \delta+\sup _{I_{z}(\tau)} v^{\frac{1}{2}}(s, \cdot)\left[\int_{I_{z}(\tau)} \frac{\vartheta_{y}^{2}}{v \theta^{2}}(s, \xi) d \xi\right]^{\frac{1}{2}},
\end{aligned}
$$

where we have used $b_{[z]+2}(s, \tau) \in \Omega_{[z]+2}(\tau) \subset I_{z}(\tau)$. Combine (2.37) with (2.23) and (2.6) to give

$$
\theta(s, y) \lesssim 1+\sup _{I_{z}(\tau)} v(s, \cdot) \int_{I_{z}(\tau)} \frac{\vartheta_{y}^{2}}{v \theta^{2}}(s, \xi) d \xi
$$

and

$$
1-C \sup _{I_{z}(\tau)} v(s, \cdot) \int_{I_{z}(\tau)} \frac{\vartheta_{y}^{2}}{v \theta^{2}}(s, \xi) d \xi \lesssim \theta(s, y) .
$$

We plug (2.38) into (2.36) to obtain

$$
v(t, y) \lesssim 1+\int_{0}^{t} \sup _{I_{z}(\tau)} v(s, \cdot) \int_{I_{z}(\tau)} \frac{\vartheta_{y}^{2}}{v \theta^{2}}(s, \xi) d \xi d s .
$$

Taking the supremum over $I_{z}(\tau)$ with respect to $y$, we have

$$
\sup _{I_{z}(\tau)} v(t, \cdot) \lesssim 1+\int_{0}^{t} \sup _{I_{z}(\tau)} v(s, \cdot) \int_{\Omega_{i}(\tau)} \frac{\vartheta_{y}^{2}}{v \theta^{2}}(s, \xi) d \xi d s .
$$

Applying Gronwall's inequality to (2.40), we can deduce from (2.20) that

$$
\sup _{I_{z}(\tau)} v(t, \cdot) \leq C_{1} \quad \text { for all } t \in[0, \tau]
$$


where $C_{1}>0$ is some constant independent of $t, \tau$ and $z$. Noting that $z \in I_{z}(\tau)$, we deduce from (2.41) that $v(\tau, z) \leq C_{1}$. Since $(\tau, z) \in \Omega_{T}$ is arbitrary, we conclude

$$
v(\tau, z) \leq C_{1} \quad \text { for all }(\tau, z) \in \Omega_{T} .
$$

Step 3. On the other hand, in view of (2.22), (2.31) and (2.35), we integrate (2.25) on $I_{z}(\tau)$ with respect to $y$ to find

$$
1 \lesssim \int_{I_{z}(\tau)} v(t, y) d y \lesssim \mathrm{e}^{-t / C}+\int_{0}^{t} \frac{A_{z}(t)}{A_{z}(s)} d s
$$

Consequently, we have

$$
\int_{0}^{t} \frac{A_{z}(t)}{A_{z}(s)} d s \gtrsim 1-C \mathrm{e}^{-t / C} .
$$

Inserting (2.39), (2.42) and (2.43) into (2.36), we have

$$
\begin{aligned}
v(t, y) & \gtrsim \int_{0}^{t} \frac{A_{z}(t)}{A_{z}(s)} d s-C \int_{0}^{t} \frac{A_{z}(t)}{A_{z}(s)} \int_{I_{z}(\tau)} \frac{\vartheta_{y}^{2}}{v \theta^{2}} d \xi d s \\
& \gtrsim 1-C \mathrm{e}^{-t / C}-C\left(\int_{0}^{\frac{t}{2}}+\int_{\frac{t}{2}}^{t} \frac{A_{z}(t)}{A_{z}(s)} \int_{I_{z}(\tau)} \frac{\vartheta_{y}^{2}}{v \theta^{2}} d \xi d s\right. \\
& \gtrsim 1-C \mathrm{e}^{-t / C}-C \int_{0}^{\frac{t}{2}} \mathrm{e}^{-\frac{t-s}{C}} \int_{I_{z}(\tau)} \frac{\vartheta_{y}^{2}}{v \theta^{2}} d \xi d s-C \int_{\frac{t}{2}}^{t} \int_{I_{z}(\tau)} \frac{\vartheta_{y}^{2}}{v \theta^{2}} d \xi d s \\
& \gtrsim 1-C \mathrm{e}^{-t / C}-C \mathrm{e}^{-\frac{t}{2 C}}-C \int_{\frac{t}{2}}^{t} \int_{I_{z}(\tau)} \frac{\vartheta_{y}^{2}}{v \theta^{2}} d \xi d s \\
& \gtrsim 1 \text { for all }(t, y) \in\left[T_{0}, \tau\right] \times I_{z}(\tau),
\end{aligned}
$$

where $T_{0}$ is a positive constant independent of $t$. In particular, the estimate (2.44) implies

$$
v(\tau, z) \gtrsim 1 \text { for all } \tau \geq T_{0}, z>Y(\tau) .
$$

As in $[12,13]$, we can derive a positive lower bound for $v$, that is,

$$
v(\tau, z) \gtrsim e^{-C t}, \quad \text { for }(\tau, z) \in \Omega_{T} .
$$

Finally, we combine (2.46), (2.42) and (2.45) to get (2.30). This completes the proof.

As a corollary of Lemma 2.6, we get the uniform bounds for the density $\rho$ in the Eulerian coordinate.

Corollary 2.7. If (2.6) holds for a sufficiently small $\epsilon_{0}>0$, then

$$
C_{1}^{-1} \leq \rho(t, x) \leq C_{1} \quad \text { for all }(t, x) \in[0, T] \times \mathbb{R}_{+},
$$

where the positive constant $C_{1}$ depends solely on $\inf _{\mathbb{R}_{+}}\left(\rho_{0}, \theta_{0}\right)$ and $\left\|\left(\phi_{0}, \psi_{0}, \vartheta_{0}\right)\right\|_{1}$.

\subsection{Pointwise estimates of temperature}

In the following lemma, we employ the maximum principle to get the lower bound for the temperature, which does depend on the time $t$.

Lemma 2.8. Suppose that (2.6) holds for a suitably small $\epsilon_{0}>0$. Then

$$
\inf _{\mathbb{R}_{+}} \theta(t, \cdot) \geq \frac{\inf _{\mathbb{R}_{+}} \theta(s, \cdot)}{C_{2} \inf _{\mathbb{R}_{+}} \theta(s, \cdot)(t-s)+1} \quad \text { for } 0 \leq s \leq t \leq T,
$$

where the positive constant $C_{2}$ depends only upon $\inf _{\mathbb{R}_{+}}\left(\rho_{0}, \theta_{0}\right)$ and $\left\|\left(\phi_{0}, \psi_{0}, \vartheta_{0}\right)\right\|_{1}$. 
Proof. It follows from $(1.1)_{3}$ that $\theta$ satisfies

$$
\theta_{t}+u \theta_{x}-\frac{\kappa}{c_{v} \rho} \theta_{x x}=\frac{\mu}{c_{v} \rho}\left[u_{x}^{2}-\frac{P}{\mu} u_{x}\right] \geq-\frac{P^{2}}{4 \mu c_{v} \rho}=-\frac{R^{2} \rho}{4 \mu c_{v}} \theta^{2} .
$$

Hence we deduce from (2.47) that

$$
\theta_{t}+u \theta_{x}-\frac{\kappa}{c_{v} \rho} \theta_{x x}+C_{2} \theta^{2} \geq 0 .
$$

Let $\Theta:=\theta-\underline{\theta}$ with $\underline{\theta}:=\frac{\inf _{\mathbb{R}_{+}} \theta(s, \cdot)}{C_{2} \inf _{\mathbb{R}_{+}} \theta(s, \cdot)(t-s)+1}$. We observe

$$
\left.\Theta\right|_{x=0, \infty} \geq 0,\left.\quad \Theta\right|_{t=s} \geq 0,
$$

and

$$
\Theta_{t}+u \Theta_{x}-\frac{\kappa}{c_{v} \rho} \Theta_{x x}+C_{2}(\theta+\underline{\theta}) \Theta=\theta_{t}+u \theta_{x}-\frac{\kappa}{c_{v} \rho} \theta_{x x}+C_{2} \theta^{2} \geq 0 .
$$

Applying the weak maximum principle for the parabolic equation, we have that $\Theta(t, x) \geq 0$ for $0 \leq s \leq$ $t \leq T$ and $x \in \mathbb{R}_{+}$. This completes the proof of the lemma.

Next we have the $L^{2}$-norm in both time and space of $\vartheta_{x}$.

Lemma 2.9. If (2.6) holds for a sufficiently small positive constant $\epsilon_{0}$, then

$$
\sup _{0 \leq t \leq T} \int_{\mathbb{R}_{+}}\left[\phi^{2}+\vartheta^{2}+\psi^{2}\right]+\int_{0}^{T} \int_{\mathbb{R}_{+}}\left[\left(1+\theta+\psi^{2}\right) \psi_{x}^{2}+\vartheta_{x}^{2}\right] \lesssim 1 .
$$

Proof. We divide the proof into five steps.

Step 1. First, for each $t \geq 0$ and $a>0$, we denote

$$
\Omega_{a}^{\prime}(t):=\left\{x \in \mathbb{R}_{+}: \vartheta(t, x)>a\right\} .
$$

Then it follows from (2.8) and (2.47) that

$$
\begin{aligned}
& \sup _{0 \leq t \leq T}\left[\int_{\mathbb{R}_{+}} \phi^{2}+\int_{\mathbb{R}_{+} \backslash \Omega_{a}^{\prime}(t)} \vartheta^{2}+\int_{0}^{t}|\phi(s, 0)|^{2} d s\right]+\int_{0}^{T} \int_{\mathbb{R}_{+} \backslash \Omega_{a}^{\prime}(t)}\left[\psi_{x}^{2}+\vartheta_{x}^{2}\right] \\
& \leq C(a) \sup _{0 \leq t \leq T}\left[\int_{\mathbb{R}_{+}} \rho \mathcal{E}+\int_{0}^{t} \rho \Phi\left(\frac{\tilde{\rho}}{\rho}\right)(s, 0) d s\right]+C(a) \int_{0}^{T} \int_{\mathbb{R}_{+}}\left[\frac{\psi_{x}^{2}}{\theta}+\frac{\vartheta_{x}^{2}}{\theta^{2}}\right] \leq C(a) .
\end{aligned}
$$

Step 2. We now estimate the integral $\int_{0}^{T} \int_{\Omega_{a}^{\prime}(t)} \vartheta_{x}^{2}$. For this purpose, we multiply $(2.2)_{3}$ by $(\vartheta-2)_{+}:=$ $\max \{\vartheta-2,0\}$ and integrate the resulting identity over $(0, t) \times \mathbb{R}_{+}$to obtain

$$
\begin{aligned}
& \frac{c_{v}}{2} \int_{\mathbb{R}_{+}} \rho(\vartheta-2)_{+}^{2}+\kappa \int_{0}^{t} \int_{\Omega_{2}^{\prime}(s)} \vartheta_{x}^{2}+R \int_{0}^{t} \int_{\mathbb{R}_{+}} \rho \theta \psi_{x}(\vartheta-2)_{+} \\
= & \frac{c_{v}}{2} \int_{\mathbb{R}_{+}} \rho_{0}\left(\vartheta_{0}-2\right)_{+}^{2}+\int_{0}^{t} \int_{\mathbb{R}_{+}} h(\vartheta-2)_{+}+\mu \int_{0}^{t} \int_{\mathbb{R}_{+}} \psi_{x}^{2}(\vartheta-2)_{+} .
\end{aligned}
$$

To estimate the last term of (2.51), we multiply $(2.2)_{2}$ by $2 \psi(\vartheta-2)_{+}$and integrate the resulting identity over $(0, t) \times \mathbb{R}_{+}$to find

$$
\begin{aligned}
& \int_{\mathbb{R}_{+}} \psi^{2} \rho(\vartheta-2)_{+}+2 \mu \int_{0}^{t} \int_{\mathbb{R}_{+}} \psi_{x}^{2}(\vartheta-2)_{+}-\int_{0}^{t} \int_{\Omega_{2}^{\prime}(s)} \rho \psi^{2}\left(\vartheta_{t}+u \vartheta_{x}\right) \\
& =\int_{\mathbb{R}_{+}} \psi_{0}^{2} \rho_{0}\left(\vartheta_{0}-2\right)_{+}+2 R \int_{0}^{t} \int_{\mathbb{R}_{+}} \rho \theta \psi_{x}(\vartheta-2)_{+}+2 R \int_{0}^{t} \int_{\Omega_{2}^{\prime}(s)} \rho \theta \psi \vartheta_{x} \\
& \quad+2 R \int_{0}^{t} \int_{\mathbb{R}_{+}} \psi(\tilde{\rho} \tilde{\theta})_{x}(\vartheta-2)_{+}-2 \mu \int_{0}^{t} \int_{\Omega_{2}^{\prime}(s)} \psi \psi_{x} \vartheta_{x}+2 \int_{0}^{t} \int_{\mathbb{R}_{+}} g \psi(\vartheta-2)_{+} .
\end{aligned}
$$


Combining (2.52) and (2.51), we have from $(2.2)_{3}$ that

$$
\begin{aligned}
& \int_{\mathbb{R}_{+}}\left[\frac{c_{v}}{2} \rho(\vartheta-2)_{+}^{2}+\psi^{2} \rho(\vartheta-2)_{+}\right]+\kappa \int_{0}^{t} \int_{\Omega_{2}^{\prime}(s)} \vartheta_{x}^{2}+\mu \int_{0}^{t} \int_{\mathbb{R}_{+}} \psi_{x}^{2}(\vartheta-2)_{+} \\
= & \int_{\mathbb{R}_{+}}\left[\frac{c_{v}}{2} \rho_{0}\left(\vartheta_{0}-2\right)_{+}^{2}+\psi_{0}^{2} \rho_{0}\left(\vartheta_{0}-2\right)_{+}\right]+\sum_{p=1}^{6} \mathcal{J}_{p},
\end{aligned}
$$

where each term $\mathcal{J}_{p}$ in the decomposition will be defined below. We now define and estimate all the terms in the decomposition. We first consider

$$
\mathcal{J}_{1}:=R \int_{0}^{t} \int_{\mathbb{R}_{+}} \rho \theta \psi_{x}(\vartheta-2)_{+}
$$

and

$$
\mathcal{J}_{2}:=2 R \int_{0}^{t} \int_{\Omega_{2}^{\prime}(s)} \rho \theta \psi \vartheta_{x}
$$

In light of (2.47) and (2.8), we have

$$
\int_{\mathbb{R}_{+}} \psi^{2}+\int_{\Omega_{1}^{\prime}(s)} \theta \lesssim \int_{\mathbb{R}_{+}} \rho \mathcal{E} \lesssim 1
$$

From Cauchy's inequality and (2.47), we obtain

$$
\begin{aligned}
\left|\mathcal{J}_{1}\right| & \leq \epsilon \int_{0}^{t} \int_{\mathbb{R}_{+}} \psi_{x}^{2}(\vartheta-2)_{+}+C(\epsilon) \int_{0}^{t} \int_{\mathbb{R}_{+}} \theta^{2}(\vartheta-2)_{+} \\
& \leq \epsilon \int_{0}^{t} \int_{\mathbb{R}_{+}} \psi_{x}^{2}(\vartheta-2)_{+}+C(\epsilon) \int_{0}^{t} \int_{\mathbb{R}_{+}} \theta(\vartheta-1)_{+}^{2} \\
& \leq \epsilon \int_{0}^{t} \int_{\mathbb{R}_{+}} \psi_{x}^{2}(\vartheta-2)_{+}+C(\epsilon) \int_{0}^{t} \sup _{\mathbb{R}_{+}}(\vartheta-1)_{+}^{2},
\end{aligned}
$$

and

$$
\begin{aligned}
\left|\mathcal{J}_{2}\right| & \leq \epsilon \int_{0}^{t} \int_{\Omega_{2}^{\prime}(s)} \vartheta_{x}^{2}+C(\epsilon) \int_{0}^{t} \int_{\Omega_{2}^{\prime}(s)} \psi^{2} \theta^{2} \\
& \leq \epsilon \int_{0}^{t} \int_{\Omega_{2}^{\prime}(s)} \vartheta_{x}^{2}+C(\epsilon) \int_{0}^{t} \int_{\Omega_{2}^{\prime}(s)} \psi^{2}(\vartheta-1)_{+}^{2} \\
& \leq \epsilon \int_{0}^{t} \int_{\Omega_{2}^{\prime}(s)} \vartheta_{x}^{2}+C(\epsilon) \int_{0}^{t} \sup _{\mathbb{R}_{+}}(\vartheta-1)_{+}^{2} .
\end{aligned}
$$

Let us define

$$
\mathcal{J}_{3}:=\int_{0}^{t} \int_{\Omega_{2}^{\prime}(s)}\left[h(\vartheta-2)_{+}+c_{v}^{-1} h \psi^{2}+2 g \psi(\vartheta-2)_{+}\right] .
$$

It follows from (2.47) and the identity $P-\tilde{P}=R \rho \vartheta+R \tilde{\theta} \phi$ that

$$
|g| \lesssim\left|\tilde{u}_{x}\right|(\phi, \psi)|, \quad| h|\lesssim|\left(\tilde{u}_{x}, \tilde{\theta}_{x}\right)||\left(\phi, \psi, \vartheta, \psi_{x}\right) \mid .
$$

Applying Cauchy's inequality to $\mathcal{J}_{3}$ yields

$$
\begin{aligned}
\left|\mathcal{J}_{3}\right| & \lesssim \int_{0}^{t} \int_{\Omega_{2}^{\prime}(s)}\left|\left(\tilde{u}_{x}, \tilde{\theta}_{x}\right)\right|\left|\left(\phi, \psi, \vartheta, \psi_{x}\right)\right|\left[(\vartheta-2)_{+}(1+|\psi|)+\psi^{2}\right] \\
& \lesssim \int_{0}^{t} \int_{\Omega_{2}^{\prime}(s)}\left|\left(\tilde{u}_{x}, \tilde{\theta}_{x}\right)\right|\left[\left(\phi^{2}+\psi^{2}+\vartheta^{2}\right)+\psi_{x}^{2}+(\vartheta-2)_{+}^{2}\left(1+\psi^{2}\right)+\psi^{4}\right] .
\end{aligned}
$$


We obtain from (1.9) and (2.54) that

$$
\begin{aligned}
& \int_{0}^{t} \int_{\mathbb{R}_{+}}\left|\left(\tilde{u}_{x}, \tilde{\theta}_{x}\right)\right|(\vartheta-2)_{+}^{2}\left(1+\psi^{2}\right) \lesssim \int_{0}^{t} \sup _{\mathbb{R}_{+}}(\vartheta-1)_{+}^{2}, \\
& \int_{0}^{t} \int_{\mathbb{R}_{+}}\left|\left(\tilde{u}_{x}, \tilde{\theta}_{x}\right)\right| \psi^{4} \lesssim \int_{0}^{t}\|\psi\|_{L^{\infty}}^{4}\left\|\left(\tilde{u}_{x}, \tilde{\theta}_{x}\right)\right\|_{L^{1}} \lesssim \delta \int_{0}^{t}\|\psi\|^{2}\left\|\psi_{x}\right\|^{2} .
\end{aligned}
$$

Plugging (2.13), (2.59)-(2.60) into (2.58), we deduce from (2.50), (2.54) and Lemma 2.2 that

$$
\begin{aligned}
\left|\mathcal{J}_{3}\right| & \lesssim \delta \int_{0}^{t}\left[|\phi(s, 0)|^{2}+\left\|\left(\phi_{x}, \psi_{x}, \vartheta_{x}\right)(s)\right\|^{2}\right] d s+\int_{0}^{t} \sup (\vartheta-1)_{+}^{2} \\
& \lesssim 1+\delta \int_{0}^{t} \int_{\mathbb{R}_{+}}\left[m_{2}^{-1} \frac{\theta \phi_{x}^{2}}{\rho^{2}}+M_{2}^{2}\left(\frac{\psi_{x}^{2}}{\theta}+\frac{\vartheta_{x}^{2}}{\theta^{2}}\right)\right]+\int_{0}^{t} \sup _{\mathbb{R}_{+}}(\vartheta-1)_{+}^{2} \\
& \lesssim 1+\int_{0}^{t} \sup _{\mathbb{R}_{+}}(\vartheta-1)_{+}^{2} .
\end{aligned}
$$

Let us now consider the term

$$
\mathcal{J}_{4}:=2 R \int_{0}^{t} \int_{\mathbb{R}_{+}} \psi(\tilde{\rho} \tilde{\theta})_{x}(\vartheta-2)_{+}
$$

which is trivially estimated by

$$
\left|\mathcal{J}_{4}\right| \lesssim \int_{0}^{t} \sup _{\mathbb{R}_{+}}(\vartheta-2)_{+}\|\psi\|\left\|\left(\tilde{\rho}_{x}, \tilde{\theta}_{x}\right)\right\| \lesssim \int_{0}^{t} \sup _{\mathbb{R}_{+}}(\vartheta-1)_{+}^{2} .
$$

For the term

$$
\mathcal{J}_{5}:=\int_{0}^{t} \int_{\Omega_{2}^{\prime}(s)}\left[\mu \psi_{x}^{2} c_{v}^{-1} \psi^{2}-P \psi_{x} c_{v}^{-1} \psi^{2}-2 \mu \psi \psi_{x} \vartheta_{x}\right],
$$

we apply Cauchy's inequality and (2.54) to deduce

$$
\begin{aligned}
\left|\mathcal{J}_{5}\right| & \lesssim \epsilon \int_{0}^{t} \int_{\Omega_{2}^{\prime}(s)} \vartheta_{x}^{2}+C(\epsilon) \int_{0}^{t} \int_{\Omega_{2}^{\prime}(s)} \psi^{2} \psi_{x}^{2}+\int_{0}^{t} \int_{\Omega_{2}^{\prime}(s)} \psi^{2} \theta^{2} \\
& \lesssim \epsilon \int_{0}^{t} \int_{\Omega_{2}^{\prime}(s)} \vartheta_{x}^{2}+C(\epsilon) \int_{0}^{t} \int_{\Omega_{2}^{\prime}(s)} \psi^{2} \psi_{x}^{2}+\int_{0}^{t} \int_{\Omega_{2}^{\prime}(s)} \psi^{2}(\vartheta-1)_{+}^{2} \\
& \lesssim \epsilon \int_{0}^{t} \int_{\Omega_{2}^{\prime}(s)} \vartheta_{x}^{2}+C(\epsilon) \int_{0}^{t} \int_{\Omega_{2}^{\prime}(s)} \psi^{2} \psi_{x}^{2}+\int_{0}^{t} \sup _{\mathbb{R}_{+}}(\vartheta-1)_{+}^{2} .
\end{aligned}
$$

We finally consider

$$
\mathcal{J}_{6}:=\int_{0}^{t} \int_{\Omega_{2}^{\prime}(s)} c_{v}^{-1} \kappa \psi^{2} \vartheta_{x x} .
$$

In order to estimate $\mathcal{J}_{6}$, we apply Lebesgue's dominated convergence theorem to find

$$
\begin{aligned}
\mathcal{J}_{6} & =\frac{\kappa}{c_{v}} \lim _{\nu \rightarrow 0^{+}} \int_{0}^{t} \int_{\mathbb{R}_{+}} \varphi_{\nu}(\vartheta) \psi^{2} \vartheta_{x x} \\
& =\frac{\kappa}{c_{v}} \lim _{\nu \rightarrow 0^{+}} \int_{0}^{t} \int_{\mathbb{R}_{+}}\left[-2 \varphi_{\nu}(\vartheta) \psi \psi_{x} \vartheta_{x}-\varphi_{\nu}^{\prime}(\vartheta) \psi^{2} \vartheta_{x}^{2}\right] \\
& \leq-\frac{\kappa}{c_{v}} \lim _{\nu \rightarrow 0^{+}} \int_{0}^{t} \int_{\mathbb{R}_{+}} 2 \varphi_{\nu}(\vartheta) \psi \psi_{x} \vartheta_{x} \\
& \leq \epsilon \int_{0}^{t} \int_{\mathbb{R}_{+}} \vartheta_{x}^{2}+C(\epsilon) \int_{0}^{T} \int_{\mathbb{R}_{+}} \psi^{2} \psi_{x}^{2}
\end{aligned}
$$


where the approximate scheme $\varphi_{\nu}(\vartheta)$ is defined by

$$
\varphi_{\nu}(\vartheta)= \begin{cases}1, & \vartheta-2>\nu, \\ (\vartheta-2) / \nu, & 0<\vartheta-2 \leq \nu, \\ 0, & \vartheta-2 \leq 0 .\end{cases}
$$

Plugging (2.55)-(2.56), (2.61)-(2.64) into (2.53), we get from (2.47) that

$$
\begin{aligned}
& \int_{\mathbb{R}_{+}}(\vartheta-2)_{+}^{2}+\int_{0}^{t} \int_{\Omega_{2}^{\prime}(s)}\left[\vartheta_{x}^{2}+\psi_{x}^{2}(\vartheta-2)_{+}\right] \\
\lesssim & 1+\epsilon \int_{0}^{t} \int_{\mathbb{R}_{+}} \vartheta_{x}^{2}+C(\epsilon) \int_{0}^{t} \int_{\mathbb{R}_{+}} \psi^{2} \psi_{x}^{2}+C(\epsilon) \int_{0}^{t} \sup _{\mathbb{R}_{+}}(\vartheta-1)_{+}^{2} .
\end{aligned}
$$

Step 3. We obtain from (2.8) that

$$
\begin{aligned}
\int_{0}^{t} \int_{\mathbb{R}_{+}}\left[\vartheta_{x}^{2}+\psi_{x}^{2} \theta\right] & \lesssim \int_{0}^{t} \int_{\Omega_{3}^{\prime}(s)}\left[\vartheta_{x}^{2}+\psi_{x}^{2}(\vartheta-2)_{+}\right]+\int_{0}^{t} \int_{\mathbb{R}_{+} \backslash \Omega_{3}^{\prime}(s)}\left[\frac{\vartheta_{x}^{2}}{\theta^{2}}+\frac{\psi_{x}^{2}}{\theta}\right] \\
& \lesssim \int_{0}^{t} \int_{\Omega_{2}^{\prime}(s)}\left[\vartheta_{x}^{2}+\psi_{x}^{2}(\vartheta-2)_{+}\right]+1
\end{aligned}
$$

Combining (2.66) and (2.65), and choosing $\epsilon$ sufficiently small, we have

$$
\int_{\mathbb{R}_{+}}(\vartheta-2)_{+}^{2}+\int_{0}^{t} \int_{\mathbb{R}_{+}}\left[\vartheta_{x}^{2}+\psi_{x}^{2} \theta\right] \lesssim 1+\int_{0}^{t} \sup _{\mathbb{R}_{+}}(\vartheta-1)_{+}^{2}+\int_{0}^{t} \int_{\mathbb{R}_{+}} \psi^{2} \psi_{x}^{2}
$$

Step 4. To estimate the last term of (2.67), we multiply $(2.2)_{2}$ by $\psi^{3}$ and then integrate the resulting identity over $(0, t) \times \mathbb{R}_{+}$to have

$$
\begin{aligned}
& \frac{1}{4} \int_{\mathbb{R}_{+}} \rho \psi^{4}+3 \mu \int_{0}^{t} \int_{\mathbb{R}_{+}} \psi^{2} \psi_{x}^{2}-\frac{1}{4} \int_{\mathbb{R}_{+}} \rho_{0} \psi_{0}^{4} \\
= & 3 R \int_{0}^{t} \int_{\mathbb{R}_{+}} \psi^{2} \psi_{x} \tilde{\theta} \phi+3 R \int_{0}^{t} \int_{\mathbb{R}_{+}} \psi^{2} \psi_{x} \rho \vartheta+\int_{0}^{t} \int_{\mathbb{R}_{+}} g \psi^{3} .
\end{aligned}
$$

From (2.50) and (2.54), we have

$$
\begin{aligned}
& \int_{0}^{t} \int_{\mathbb{R}_{+}} \psi^{2} \psi_{x} \tilde{\theta} \phi+\int_{0}^{t} \int_{\mathbb{R}_{+} \backslash \Omega_{2}^{\prime}(s)} \psi^{2} \psi_{x} \rho \vartheta \\
& \lesssim \int_{0}^{t}\|\psi\|_{L_{x}^{\infty}}^{2}\left\|\psi_{x}\right\|\left[\|\phi\|+\|\vartheta\|_{\left.L^{2}\left(\mathbb{R}_{+} \backslash \Omega_{2}^{\prime}(s)\right)\right] \lesssim \int_{0}^{t}\left\|\psi_{x}\right\|^{2}}\right.
\end{aligned}
$$

We then apply Cauchy's inequality to derive

$$
\begin{aligned}
\int_{0}^{t} \int_{\Omega_{2}^{\prime}(s)} \psi^{2} \psi_{x} \rho \vartheta & \leq \epsilon \int_{0}^{t} \int_{\mathbb{R}_{+}} \psi^{2} \psi_{x}^{2}+C(\epsilon) \int_{0}^{t} \int_{\Omega_{2}^{\prime}(s)} \psi^{2} \vartheta^{2} \\
& \leq \epsilon \int_{0}^{t} \int_{\mathbb{R}_{+}} \psi^{2} \psi_{x}^{2}+C(\epsilon) \int_{0}^{t} \sup _{\mathbb{R}_{+}}(\vartheta-1)_{+}^{2}
\end{aligned}
$$


In view of (2.50), (2.54), (2.57) and (2.7), we have

$$
\begin{aligned}
\int_{0}^{t} \int_{\mathbb{R}_{+}} g \psi^{3} & \lesssim \int_{0}^{t} \int_{\mathbb{R}_{+}}\left|\tilde{u}_{x}\right|\left(\psi^{4}+\phi^{4}\right) \\
& \lesssim \delta \int_{0}^{t}\left(\|\psi\|^{2}\left\|\psi_{x}\right\|^{2}+\|\phi\|^{2}\left\|\phi_{x}\right\|^{2}\right) \\
& \lesssim \delta \int_{0}^{t}\left\|\psi_{x}\right\|^{2}+\delta M_{1}^{2} m_{2}^{-1} \int_{0}^{t} \int_{\mathbb{R}_{+}} \frac{\theta \phi_{x}^{2}}{\rho^{2}} \\
& \lesssim \delta \int_{0}^{t}\left\|\psi_{x}\right\|^{2}+\delta M_{1}^{2} m_{2}^{-1} M_{2}
\end{aligned}
$$

Plugging (2.69) -(2.71) into (2.68), and taking $\epsilon$ sufficiently small, we derive from (2.6) that

$$
\int_{\mathbb{R}_{+}} \psi^{4}+\int_{0}^{t} \int_{\mathbb{R}_{+}} \psi^{2} \psi_{x}^{2} \lesssim 1+\int_{0}^{t} \int_{\mathbb{R}_{+}} \psi_{x}^{2}+\int_{0}^{t} \sup _{\mathbb{R}_{+}}(\vartheta-1)_{+}^{2} .
$$

We note from (2.8) that

$$
\int_{0}^{t} \int_{\mathbb{R}_{+}} \psi_{x}^{2} \leq \epsilon \int_{0}^{t} \int_{\mathbb{R}_{+}} \theta \psi_{x}^{2}+C(\epsilon) \int_{0}^{t} \int_{\mathbb{R}_{+}} \frac{\psi_{x}^{2}}{\theta} \leq \epsilon \int_{0}^{t} \int_{\mathbb{R}_{+}} \theta \psi_{x}^{2}+C(\epsilon) .
$$

Combination of (2.73) and (2.72) yields

$$
\int_{\mathbb{R}_{+}} \psi^{4}+\int_{0}^{t} \int_{\mathbb{R}_{+}}\left(1+\psi^{2}\right) \psi_{x}^{2} \lesssim C(\epsilon)+\epsilon \int_{0}^{t} \int_{\mathbb{R}_{+}} \theta \psi_{x}^{2}+\int_{0}^{t} \sup _{\mathbb{R}_{+}}(\vartheta-1)_{+}^{2} .
$$

We plug (2.74) into (2.67) and choose $\epsilon$ suitable small to find

$$
\int_{\mathbb{R}_{+}}\left[(\vartheta-2)_{+}^{2}+\psi^{4}\right]+\int_{0}^{t} \int_{\mathbb{R}_{+}}\left[\vartheta_{x}^{2}+\psi_{x}^{2}\left(1+\theta+\psi^{2}\right)\right] \lesssim 1+\int_{0}^{t} \sup _{\mathbb{R}_{+}}(\vartheta-1)_{+}^{2} .
$$

Step 5. It remains to estimate the last term of (2.75). According to the fundamental theorem of calculus, we have from (2.54) that

$$
\begin{aligned}
\int_{0}^{T} \sup _{\mathbb{R}_{+}}(\vartheta-1)_{+}^{2} & \leq \int_{0}^{T}\left[\int_{\Omega_{1}^{\prime}(s)}\left|\vartheta_{x}\right|\right]^{2} \\
& \leq \int_{0}^{T}\left[\int_{\Omega_{1}^{\prime}(s)} \frac{\vartheta_{x}^{2}}{\theta} \int_{\Omega_{1}^{\prime}(s)} \theta\right] \\
& \leq \epsilon \int_{0}^{T} \int_{\mathbb{R}_{+}} \vartheta_{x}^{2}+C(\epsilon) \int_{0}^{T} \int_{\mathbb{R}_{+}} \frac{\vartheta_{x}^{2}}{\theta^{2}} \\
& \leq \epsilon \int_{0}^{T} \int_{\mathbb{R}_{+}} \vartheta_{x}^{2}+C(\epsilon) .
\end{aligned}
$$

Plug (2.76) into (2.75) and choose $\epsilon>0$ suitable small to obtain (2.49). This completes the proof of the lemma.

We obtain the upper bound for the temperature uniformly in both time and space in the next lemma, by combining Lemma 2.9 and some desired uniform estimates on the spatial derivatives of $(\phi, \psi, \vartheta)$.

Lemma 2.10. If (2.6) holds for a sufficiently small $\epsilon>0$, then we have

$$
\begin{gathered}
\theta(t, x) \leq C_{3} \quad \text { for all }(t, x) \in[0, T] \times \mathbb{R}_{+}, \\
\sup _{0 \leq t \leq T}\|(\phi, \psi, \vartheta)(t)\|_{1}^{2}+\int_{0}^{T}\left[\left\|\sqrt{\theta} \phi_{x}(t)\right\|^{2}+\left\|\left(\psi_{x}, \vartheta_{x}\right)(t)\right\|_{1}^{2}\right] d t \leq C_{4}^{2} .
\end{gathered}
$$


Proof. First, plugging (2.47) into (2.17), we deduce

$$
\int_{\mathbb{R}_{+}} \phi_{x}^{2}+\int_{0}^{t} \int_{\mathbb{R}_{+}} \theta \phi_{x}^{2} \lesssim 1+\int_{0}^{t} \int_{\mathbb{R}_{+}}\left[\psi_{x}^{2}+\vartheta_{x}^{2}+\frac{\vartheta_{x}^{2}}{\theta^{2}}\right] \lesssim 1
$$

where we employed (2.8) and (2.49) in the last inequality.

Next, multiply $(2.2)_{2}$ by $\frac{\psi_{x x}}{\rho}$ to derive

$$
\left(\frac{\psi_{x}^{2}}{2}\right)_{t}-\left[\psi_{t} \psi_{x}+\frac{1}{2} u \psi_{x}^{2}\right]_{x}+\frac{1}{2} u_{x} \psi_{x}^{2}+\mu \frac{\psi_{x x}^{2}}{\rho}=\frac{(P-\tilde{P})_{x}}{\rho} \psi_{x x}-\frac{g}{\rho} \psi_{x x}
$$

Integrating this last identity over $(0, T) \times \mathbb{R}_{+}$, we obtain from (2.47) and Cauchy's inequality that

$$
\begin{aligned}
& \int_{\mathbb{R}_{+}} \psi_{x}^{2}-\int_{0}^{T} \psi_{x}^{2}(t, 0) d t+\int_{0}^{T} \int_{\mathbb{R}_{+}} \psi_{x x}^{2} \\
& \lesssim 1+\int_{0}^{T} \int_{\mathbb{R}_{+}}\left[(P-\tilde{P})_{x}^{2}+g^{2}+\left|\tilde{u}_{x}\right| \psi_{x}^{2}+\left|\psi_{x}\right|^{3}\right] .
\end{aligned}
$$

Apply Sobolev's inequality and (2.49) to obtain

$$
\begin{aligned}
\int_{0}^{T} \psi_{x}^{2}(t, 0) d t+\int_{0}^{T} \int_{\mathbb{R}_{+}}\left|\psi_{x}\right|^{3} & \lesssim \int_{0}^{T}\left\|\psi_{x}\right\|\left\|\psi_{x x}\right\|+\int_{0}^{T}\left\|\psi_{x}\right\|\left\|^{\frac{5}{2}}\right\| \psi_{x x} \|^{\frac{1}{2}} \\
& \lesssim \epsilon \int_{0}^{T} \int_{\mathbb{R}_{+}} \psi_{x x}^{2}+C(\epsilon) \int_{0}^{T}\left[\left\|\psi_{x}\right\|^{2}+\left\|\psi_{x}\right\|^{\frac{10}{3}}\right] \\
& \lesssim C(\epsilon)\left[1+\sup _{0 \leq t \leq T}\left\|\psi_{x}\right\|^{\frac{4}{3}}\right]+\epsilon \int_{0}^{T} \int_{\mathbb{R}_{+}} \psi_{x x}^{2} .
\end{aligned}
$$

In view of the identity $(P-\tilde{P})_{x}=R\left(\theta \phi_{x}+\rho \vartheta_{x}+\phi \tilde{\theta}_{x}+\vartheta \tilde{\rho}_{x}\right),(2.57),(2.79),(2.49)$ and $(2.13)$, we derive

$$
\begin{aligned}
& \int_{0}^{T} \int_{\mathbb{R}_{+}}\left[(P-\tilde{P})_{x}^{2}+g^{2}+\left|\tilde{u}_{x}\right| \psi_{x}^{2}\right] \\
& \lesssim \int_{0}^{T} \int_{\mathbb{R}_{+}}\left[\left|\left(\theta \phi_{x}, \psi_{x}, \vartheta_{x}\right)\right|^{2}+\left|\left(\tilde{\rho}_{x}, \tilde{u}_{x}, \tilde{\theta}_{x}\right)\right|^{2}|(\phi, \psi, \vartheta)|^{2}\right] \\
& \lesssim 1+\|\theta\|_{L^{\infty}\left([0, T] \times \mathbb{R}_{+}\right)} .
\end{aligned}
$$

We plug (2.81)-(2.82) into (2.80) to get

$$
\sup _{0 \leq t \leq T}\left\|\psi_{x}(t)\right\|^{2}+\int_{0}^{T} \int_{\mathbb{R}_{+}} \psi_{x x}^{2} \lesssim 1+\|\theta\|_{L^{\infty}\left([0, T] \times \mathbb{R}_{+}\right)}+\sup _{0 \leq t \leq T}\left\|\psi_{x}\right\|^{\frac{4}{3}} .
$$

Then Young's inequality yields the estimate

$$
\sup _{0 \leq t \leq T}\left\|\psi_{x}(t)\right\|^{2}+\int_{0}^{T} \int_{\mathbb{R}_{+}} \psi_{x x}^{2} \lesssim 1+\|\theta\|_{L^{\infty}\left([0, T] \times \mathbb{R}_{+}\right)} .
$$

Next, multiply $(2.2)_{3}$ by $\frac{\vartheta_{x x}}{\rho}$ and integrate the resulting identity over $(0, T) \times \mathbb{R}_{+}$to have

$$
\frac{c_{v}}{2} \int_{\mathbb{R}_{+}} \vartheta_{x}^{2}+\kappa \int_{0}^{T} \int_{\mathbb{R}_{+}} \frac{\vartheta_{x x}^{2}}{\rho}=\frac{c_{v}}{2} \int_{\mathbb{R}_{+}} \vartheta_{0 x}^{2}+\int_{0}^{T} \int_{\mathbb{R}_{+}}\left[c_{v} u \vartheta_{x}-\mu \frac{\psi_{x}^{2}}{\rho}+R \theta \psi_{x}-\frac{h}{\rho}\right] \vartheta_{x x}
$$


which combined with (2.47) implies

$$
\begin{aligned}
\int_{\mathbb{R}_{+}} \vartheta_{x}^{2}+\int_{0}^{T} \int_{\mathbb{R}_{+}} \vartheta_{x x}^{2} \lesssim & 1+\int_{0}^{T} \int_{\mathbb{R}_{+}}\left[u^{2} \vartheta_{x}^{2}+\left\|\psi_{x}\right\|_{L^{\infty}}^{2} \psi_{x}^{2}+\theta^{2} \psi_{x}^{2}+h^{2}\right] \\
\lesssim & 1+\int_{0}^{T}\left(1+\|\psi\|\left\|\psi_{x}\right\|\right)\left\|\vartheta_{x}\right\|^{2}+\int_{0}^{T}\left\|\psi_{x}\right\|^{3}\left\|\psi_{x x}\right\| \\
& +\|\theta\|_{L^{\infty}\left([0, T] \times \mathbb{R}_{+}\right)} \int_{0}^{T} \int_{\mathbb{R}_{+}} \theta \psi_{x}^{2}+\int_{0}^{T} \int_{\mathbb{R}_{+}} h^{2} .
\end{aligned}
$$

By (2.57), (2.13) and (2.83), we have $\int_{0}^{T} \int_{\mathbb{R}_{+}} h^{2} \lesssim 1$, and

$$
\int_{0}^{T}\left\|\psi_{x}\right\|^{3}\left\|\psi_{x x}\right\| \lesssim \sup _{0 \leq t \leq T}\left\|\psi_{x}\right\|^{2} \int_{0}^{T}\left(\left\|\psi_{x}\right\|^{2}+\left\|\psi_{x x}\right\|^{2}\right) \lesssim 1+\|\theta\|_{L^{\infty}\left([0, T] \times \mathbb{R}_{+}\right)}^{2} .
$$

In light of (2.79), (2.83) and (2.49), we then obtain

$$
\int_{\mathbb{R}_{+}} \vartheta_{x}^{2}+\int_{0}^{T} \int_{\mathbb{R}_{+}} \vartheta_{x x}^{2} \lesssim 1+\|\theta\|_{L^{\infty}\left([0, T] \times \mathbb{R}_{+}\right)}^{2} .
$$

Finally, it follows from (2.49) and (2.85) that

$$
\|\theta-\tilde{\theta}\|_{L^{\infty}\left([0, T] \times \mathbb{R}_{+}\right)}^{2} \sup _{0 \leq t \leq T}\|\vartheta(t)\|\left\|\vartheta_{x}(t)\right\| \lesssim 1+\|\theta\|_{L^{\infty}\left([0, T] \times \mathbb{R}_{+}\right)} .
$$

This implies (2.77) by virtue of Cauchy's inequality. Combine (2.80), (2.83) and (2.85) to give

$$
\sup _{0 \leq t \leq T} \int_{\mathbb{R}_{+}}\left[\phi_{x}^{2}+\psi_{x}^{2}+\vartheta_{x}^{2}\right]+\int_{0}^{T} \int_{\mathbb{R}_{+}}\left[\theta \phi_{x}^{2}+\psi_{x x}^{2}+\vartheta_{x x}^{2}\right] \lesssim 1,
$$

which together with (2.49) yields (2.78). This completes the proof of the lemma.

\section{Proof of Theorem 1}

In this section, we prove Theorem 1 in six steps by employing the continuation argument.

Step 1. Set $T_{1}=128 \lambda_{3}^{-4} C_{4}^{4}$. We choose positive constants $\Pi, \lambda_{i}$, and $\Lambda_{i}(i=1,2,3)$ such that $\left\|\left(\phi_{0}, \psi_{0}, \vartheta_{0}\right)\right\|_{1} \leq \Pi$ and

$$
\lambda_{1} \leq \rho_{0}(x) \leq \Lambda_{1}, \quad \lambda_{2} \leq \theta_{0}(x) \leq \Lambda_{2}, \quad \lambda_{3} \leq \tilde{\theta}(x) \leq \Lambda_{3} \quad \text { for all } x \in \mathbb{R}_{+} .
$$

Applying Proposition 2.1, we see that there exists a constant $0<t_{1} \leq \min \left\{T_{1}, T_{0}\left(\lambda_{1}, \lambda_{2}, \Pi\right)\right\}$ such that the problem (2.2) has a unique solution $(\phi, \psi, \vartheta) \in X\left(0, t_{1} ; \frac{1}{2} \lambda_{1}, 2 \Lambda_{1} ; \frac{1}{2} \lambda_{2}, 2 \Lambda_{2}\right)$.

Let $0<\delta \leq \delta_{1}$ with

$$
\Xi\left(\frac{\lambda_{1}}{2}, 2 \Lambda_{1}, \frac{\lambda_{2}}{2}, 2 \Lambda_{2}\right) \delta_{1}=\epsilon_{0} .
$$

Then we can apply Lemmas 2.6, 2.8 and 2.10 with $T=t_{1}$ to obtain that the local solution $(\phi, \psi, \vartheta)$ constructed above satisfies for each $t \in\left[0, t_{1}\right]$ that

$$
\theta(t, x) \geq \frac{\lambda_{2}}{C_{2} \lambda_{2} T_{1}+1} \quad \text { for all } x \in \mathbb{R}_{+},
$$

and

$$
\begin{gathered}
C_{1}^{-1} \leq \rho(t, x) \leq C_{1}, \quad \theta(t, x) \leq C_{3} \quad \text { for all } x \in \mathbb{R}_{+}, \\
\|(\phi, \psi, \vartheta)(t)\|_{1}^{2}+\int_{0}^{t}\left[\left\|\sqrt{\theta} \phi_{x}(s)\right\|^{2}+\left\|\left(\psi_{x}, \vartheta_{x}\right)(s)\right\|_{1}^{2}\right] d s \leq C_{4}^{2} .
\end{gathered}
$$


Step 2. If we take $(\phi, \psi, \vartheta)\left(t_{1}, \cdot\right)$ as the initial data, we can apply Proposition 2.1 and extend the local solution $(\phi, \psi, \vartheta)$ to the time interval $\left[0, t_{1}+t_{2}\right]$ with $t_{2} \leq \min \left\{T_{1}-t_{1}, T_{0}\left(\frac{1}{C_{1}}, \frac{\lambda_{2}}{C_{2} \lambda_{2} T_{1}+1}, C_{4}\right)\right\}$. Moreover, we have

$$
\frac{1}{2 C_{1}} \leq \rho(t, x) \leq 2 C_{1}, \quad \frac{\lambda_{2}}{2\left(C_{2} \lambda_{2} T_{1}+1\right)} \leq \theta(t, x) \leq 2 C_{3}
$$

for all $(t, x) \in\left[t_{1}, t_{1}+t_{2}\right] \times \mathbb{R}_{+}$. Take $0<\delta \leq \min \left\{\delta_{1}, \delta_{2}\right\}$ with

$$
\Xi\left(\frac{1}{2 C_{1}}, 2 C_{1}, \frac{\lambda_{2}}{2\left(C_{2} \lambda_{2} T_{1}+1\right)}, 2 C_{3}\right) \delta_{2}=\epsilon_{0} .
$$

Then we can employ Lemmas 2.6, 2.8 and 2.10 with $T=t_{1}+t_{2}$ that the local solution $(\phi, \psi, \vartheta)$ satisfies (3.1) and (3.2) for each $t \in\left[0, t_{1}+t_{2}\right]$.

Step 3. We repeat the argument in Step 2, to extend our solution $(\phi, \psi, \vartheta)$ to the time interval $\left[0, t_{1}+t_{2}+\right.$ $\left.t_{3}\right]$ with $t_{3} \leq \min \left\{T_{1}-\left(t_{1}+t_{2}\right), T_{0}\left(\frac{1}{C_{1}}, \frac{\lambda_{2}}{C_{2} \lambda_{2} T_{1}+1}, C_{4}\right)\right\}$. Assume that $0<\delta \leq \min \left\{\delta_{1}, \delta_{2}\right\}$. Continuing, after finitely many steps we construct the unique solution $(\phi, \psi, \vartheta)$ existing on $\left[0, T_{1}\right]$ and satisfying (3.1) and (3.2) for each $t \in\left[0, T_{1}\right]$.

Step 4. Since $T_{1} \geq 128 \lambda_{3}^{-4} C_{4}^{4}$ and

$$
\sup _{0 \leq t \leq T_{1}}\|\vartheta(t)\|_{1}^{2}+\int_{\frac{T_{1}}{2}}^{T_{1}}\left\|\vartheta_{x}(t)\right\|_{1}^{2} d t \leq C_{4}^{2}
$$

we can find a $t_{0}^{\prime} \in\left[T_{1} / 2, T_{1}\right]$ such that

$$
\left\|\vartheta\left(t_{0}^{\prime}\right)\right\| \leq C_{4}, \quad\left\|\vartheta_{x}\left(t_{0}^{\prime}\right)\right\| \leq \frac{\lambda_{3}^{2}}{8 C_{4}} .
$$

Sobolev's inequality yields

$$
\left\|\vartheta\left(t_{0}^{\prime}\right)\right\|_{L^{\infty}} \leq \sqrt{2}\left\|\vartheta\left(t_{0}^{\prime}\right)\right\|^{\frac{1}{2}}\left\|\vartheta_{x}\left(t_{0}^{\prime}\right)\right\|^{\frac{1}{2}} \leq \frac{\lambda_{3}}{2}
$$

and so

$$
\theta\left(t_{0}^{\prime}, x\right) \geq \tilde{\theta}(x)-\left\|\vartheta\left(t_{0}^{\prime}\right)\right\|_{L^{\infty}} \geq \frac{\lambda_{3}}{2} \quad \text { for all } x \in \mathbb{R}_{+} .
$$

We note here that

$$
\left\|(\phi, \psi, \vartheta)\left(t_{0}^{\prime}\right)\right\|_{1} \leq C_{4}, \quad C_{1}^{-1} \leq \rho\left(t_{0}^{\prime}, x\right) \leq C_{1}, \quad \theta\left(t_{0}^{\prime}, x\right) \leq C_{3} \quad \text { for all } x \in \mathbb{R}_{+} .
$$

Now we apply Proposition 2.1 again by taking $(\phi, \psi, \vartheta)\left(t_{0}^{\prime}, \cdot\right)$ as the initial data. Then we see that the solution $(\phi, \psi, \vartheta)$ exists on $\left[t_{0}^{\prime}, t_{0}^{\prime}+t_{1}^{\prime}\right]$ with $t_{1}^{\prime} \leq \min \left\{T_{1}, T_{0}\left(\frac{1}{C_{1}}, \frac{1}{2} \lambda_{3}, C_{4}\right)\right\}$ and satisfies

$$
\frac{1}{2 C_{1}} \leq \rho(t, x) \leq 2 C_{1}, \quad \frac{\lambda_{3}}{4} \leq \theta(t, x) \leq 2 C_{3}
$$

for all $(t, x) \in\left[t_{0}^{\prime}, t_{0}^{\prime}+t_{1}^{\prime}\right] \times \mathbb{R}_{+}$. If we take $0<\delta \leq \min \left\{\delta_{1}, \delta_{2}, \delta_{3}\right\}$ with

$$
\Xi\left(\frac{1}{2 C_{1}}, 2 C_{1}, \frac{\lambda_{3}}{4}, 2 C_{3}\right) \delta_{3}=\epsilon_{0},
$$

then we can deduce from Lemmas 2.6, 2.8 and 2.10 with $T=t_{0}^{\prime}+t_{1}^{\prime}$ that for each time $t \in\left[t_{0}^{\prime}, t_{0}^{\prime}+t_{1}^{\prime}\right]$, the local solution $(\phi, \psi, \vartheta)$ satisfies $(3.2)$ and

$$
\theta(t, x) \geq \frac{\inf _{\mathbb{R}_{+}} \theta\left(t_{0}^{\prime}, \cdot\right)}{C_{2} \inf _{\mathbb{R}_{+}} \theta\left(t_{0}^{\prime}, \cdot\right) T_{1}+1} \geq \frac{\lambda_{3}}{C_{2} \lambda_{3} T_{1}+2} \quad \text { for all } x \in \mathbb{R}_{+} .
$$

Step 5. Next if we take $(\phi, \psi, \vartheta)\left(t_{0}^{\prime}+t_{1}^{\prime}, \cdot\right)$ as the initial data, we apply Proposition 2.1 and construct the solution $(\phi, \psi, \vartheta)$ existing on the time interval $\left[0, t_{0}^{\prime}+t_{1}^{\prime}+t_{2}^{\prime}\right]$ with $t_{2}^{\prime} \leq \min \left\{T_{1}-t_{1}^{\prime}, T_{0}\left(\frac{1}{C_{1}}, \frac{\lambda_{3}}{C_{2} \lambda_{3} T_{1}+2}, C_{4}\right)\right\}$ and satisfying

$$
\frac{1}{2 C_{1}} \leq \rho(t, x) \leq 2 C_{1}, \quad \frac{\lambda_{3}}{2\left(C_{2} \lambda_{3} T_{1}+2\right)} \leq \theta(t, x) \leq 2 C_{3}
$$


for all $(t, x) \in\left[t_{0}^{\prime}+t_{1}^{\prime}, t_{0}^{\prime}+t_{1}^{\prime}+t_{2}^{\prime}\right] \times \mathbb{R}_{+}$. Let $0<\delta \leq \min \left\{\delta_{1}, \delta_{2}, \delta_{3}, \delta_{4}\right\}$ with

$$
\Xi\left(\frac{1}{2 C_{1}}, 2 C_{1}, \frac{\lambda_{3}}{2\left(C_{2} \lambda_{3} T_{1}+2\right)}, 2 C_{3}\right) \delta_{4}=\epsilon_{0} .
$$

Then we infer from Lemmas 2.6, 2.8 and 2.10 with $T=t_{0}^{\prime}+t_{1}^{\prime}+t_{2}^{\prime}$ that the local solution $(\phi, \psi, \vartheta)$ satisfies (3.3) and (3.2) for each $t \in\left[t_{0}^{\prime}, t_{0}^{\prime}+t_{1}^{\prime}+t_{2}^{\prime}\right]$. By assuming $0<\delta \leq \min \left\{\delta_{1}, \delta_{2}, \delta_{3}, \delta_{4}\right\}$, we can repeatedly apply the argument above to extend the local solution to the time interval $\left[0, t_{0}^{\prime}+T_{1}\right]$. Furthermore, we deduce that (3.3) and (3.2) hold for each $t \in\left[t_{0}^{\prime}, t_{0}^{\prime}+T_{1}\right]$. In view of $t_{0}^{\prime}+T_{1} \geq \frac{3}{2} T_{1}$, we have shown that the problem $(2.2)$ admits a unique solution $(\phi, \psi, \vartheta)$ on $\left[0, \frac{3}{2} T_{1}\right]$.

Step 6. We take $0<\delta \leq \min \left\{\delta_{1}, \delta_{2}, \delta_{3}, \delta_{4}\right\}$. As in Steps 4 and 5 , we can find $t_{0}^{\prime \prime} \in\left[t_{0}^{\prime}+T_{1} / 2, t_{0}^{\prime}+T_{1}\right]$ such that the problem $(2.2)$ admits a unique solution $(\phi, \psi, \vartheta)$ on $\left[0, t_{0}^{\prime \prime}+T_{1}\right]$, which satisfies (3.3) and (3.2) for each $t \in\left[t_{0}^{\prime}, t_{0}^{\prime \prime}+T_{1}\right]$. Since $t_{0}^{\prime \prime}+T_{1} \geq t_{0}^{\prime}+\frac{3}{2} T_{1} \geq 2 T_{1}$, we have extended the local solution $(\phi, \psi, \vartheta)$ to $\left[0,2 T_{1}\right]$. Repeating the above procedure, we can then extend the solution $(\phi, \psi, \vartheta)$ step by step to a global one provided that $\delta \leq \min \left\{\delta_{1}, \delta_{2}, \delta_{3}, \delta_{4}\right\}$. Choosing $\epsilon_{1}=\min \left\{\delta_{1}, \delta_{2}, \delta_{3}, \delta_{4}\right\}$, we then derive that the problem $(2.2)$ has a unique solution $(\phi, \psi, \vartheta)$ satisfying (3.2) and

$$
\inf _{\mathbb{R}_{+}} \theta(t, \cdot) \geq \min \left\{\frac{\lambda_{2}}{C_{2} \lambda_{2} T_{1}+1}, \frac{\lambda_{3}}{C_{2} \lambda_{3} T_{1}+2}\right\}
$$

for each $t \in[0, \infty)$.

Therefore, we can find constant $C_{5}$ depending only on $\inf _{\mathbb{R}_{+}}\left(\rho_{0}, \theta_{0}\right)$ and $\left\|\left(\phi_{0}, \psi_{0}, \vartheta_{0}\right)\right\|_{1}$ such that

$$
\sup _{0 \leq t<\infty}\|(\phi, \psi, \vartheta)(t)\|_{1}^{2}+\int_{0}^{\infty}\left[\left\|\phi_{x}(t)\right\|^{2}+\left\|\left(\psi_{x}, \vartheta_{x}\right)(t)\right\|_{1}^{2}\right] d t \leq C_{5}^{2},
$$

from which the large-time behavior (1.13) follows in a standard argument. This completes the proof of Theorem 1.

\section{Acknowledgement}

The authors express much gratitude to Professor Huijiang Zhao for his support and advice.

\section{References}

[1] L. FAn, H. Liu, T. WANG, AND H. ZhaO, Inflow problem for the one-dimensional compressible Navier-Stokes equations under large initial perturbation, J. Differential Equations, 257 (2014), pp. 3521-3553.

[2] F. Huang, J. LI, And X. Shi, Asymptotic behavior of solutions to the full compressible NavierStokes equations in the half space, Commun. Math. Sci., 8 (2010), pp. 639-654.

[3] F. Huang, A. Matsumura, And X. Shi, Viscous shock wave and boundary layer solution to an inflow problem for compressible viscous gas, Comm. Math. Phys., 239 (2003), pp. 261-285.

[4] — On the stability of contact discontinuity for compressible Navier-Stokes equations with free boundary, Osaka J. Math., 41 (2004), pp. 193-210.

[5] F. Huang And X. Qin, Stability of boundary layer and rarefaction wave to an outflow problem for compressible Navier-Stokes equations under large perturbation, J. Differential Equations, 246 (2009), pp. 4077-4096.

[6] S. JIANG, Large-time behavior of solutions to the equations of a one-dimensional viscous polytropic ideal gas in unbounded domains, Comm. Math. Phys., 200 (1999), pp. 181-193. 
[7] — Remarks on the asymptotic behaviour of solutions to the compressible Navier-Stokes equations in the half-line, Proc. Roy. Soc. Edinburgh Sect. A, 132 (2002), pp. 627-638.

[8] S. Kawashima, T. Nakamura, S. Nishibata, And P. Zhu, Stationary waves to viscous heatconductive gases in half-space: existence, stability and convergence rate, Math. Models Methods Appl. Sci., 20 (2010), pp. 2201-2235.

[9] S. Kawashima, S. Nishibata, And P. Zhu, Asymptotic stability of the stationary solution to the compressible Navier-Stokes equations in the half space, Comm. Math. Phys., 240 (2003), pp. 483-500.

[10] S. KaWASHIma AND P. ZHU, Asymptotic stability of nonlinear wave for the compressible NavierStokes equations in the half space, J. Differential Equations, 244 (2008), pp. 3151-3179.

[11] _ Asymptotic stability of rarefaction wave for the Navier-Stokes equations for a compressible fluid in the half space, Arch. Ration. Mech. Anal., 194 (2009), pp. 105-132.

[12] A. V. Kazhikhov, On the Cauchy problem for the equations of a viscous gas, Sibirsk. Mat. Zh., 23 (1982), pp. 60-64, 220.

[13] A. V. Kazhikhov And V. V. Shelukhin, Unique global solution with respect to time of initialboundary value problems for one-dimensional equations of a viscous gas, Prikl. Mat. Meh., 41 (1977), pp. 282-291.

[14] J. Li AND Z. LIANG, Some uniform estimates and large-time behavior for one-dimensional compressible navier-stokes system in unbounded domains with large data, Preprint, 2014. arXiv:1404.2214.

[15] A. Matsumura, Inflow and outflow problems in the half space for a one-dimensional isentropic model system of compressible viscous gas, Methods Appl. Anal., 8 (2001), pp. 645-666. IMS Conference on Differential Equations from Mechanics (Hong Kong, 1999).

[16] A. Matsumura And M. Mei, Convergence to travelling fronts of solutions of the p-system with viscosity in the presence of a boundary, Arch. Ration. Mech. Anal., 146 (1999), pp. 1-22.

[17] A. Matsumura And K. Nishihara, Global asymptotics toward the rarefaction wave for solutions of viscous p-system with boundary effect, Quart. Appl. Math., 58 (2000), pp. 69-83.

[18] - Large-time behaviors of solutions to an inflow problem in the half space for a one-dimensional system of compressible viscous gas, Comm. Math. Phys., 222 (2001), pp. 449-474.

[19] T. Nakamura, S. Nishibata, And T. Yuge, Convergence rate of solutions toward stationary solutions to the compressible Navier-Stokes equation in a half line, J. Differential Equations, 241 (2007), pp. 94-111.

[20] Y. Nikkuni And S. Kawashima, Stability of stationary solutions to the half-space problem for the discrete Boltzmann equation with multiple collisions, Kyushu J. Math., 54 (2000), pp. 233-255.

[21] K. Nishinara, T. Yang, and H. ZhaO, Nonlinear stability of strong rarefaction waves for compressible Navier-Stokes equations, SIAM J. Math. Anal., 35 (2004), pp. 1561-1597 (electronic).

[22] X. QIN, Large-time behaviour of solutions to the outflow problem of full compressible Navier-Stokes equations, Nonlinearity, 24 (2011), pp. 1369-1394.

[23] X. QIN AND Y. WANG, Stability of wave patterns to the inflow problem of full compressible NavierStokes equations, SIAM J. Math. Anal., 41 (2009), pp. 2057-2087.

[24] - Large-time behavior of solutions to the inflow problem of full compressible Navier-Stokes equations, SIAM J. Math. Anal., 43 (2011), pp. 341-366. 